\title{
Lipidomic profiling reveals lipid regulation by a novel LSD1 inhibitor treatment
}

\author{
YAN LI $^{1,2^{*}}$, XINYING QIAN ${ }^{2 *}$, YIYUN LIN ${ }^{2}$, LEI TAO $^{2}$, ZEPING ZUO $^{2}$, HUAQIN ZHANG $^{2}$, \\ SHENGYONG YANG ${ }^{2},{\mathrm{XIAOBO} \mathrm{CEN}^{3} \text { and YINGLAN ZHAO }}^{2}$
}

\begin{abstract}
${ }^{1}$ Department of Pharmacology, Shanxi Medical University, Taiyuan, Shanxi 030001; ${ }^{2}$ State Key Laboratory of Biotherapy and Cancer Center, West China Hospital, West China Medical School, and Collaborative Innovation Center for Biotherapy, Sichuan University; ${ }^{3}$ National Chengdu Center for Safety Evaluation of Drugs, State Key Laboratory of Biotherapy and Cancer Center, West China Hospital, Sichuan University, Collaborative Innovation Center for Biotherapy,
\end{abstract} Chengdu, Sichuan 610041, P.R. China

Received October 2, 2019; Accepted June 15, 2021

DOI: 10.3892/or.2021.8184

\begin{abstract}
Lipid metabolic alterations are associated with cancer progression. Lysine-specific demethylase 1 (LSD1) plays a crucial role in cancer and has become a promising target for cancer therapy. However, the effect of LSD1 on lipid metabolism remains unclear. In the present study, we used a LC-MS/MS-based lipidomics approach to investigate the impact of LSD1 on cancer cell lipid metabolism using ZY0511, a specific LSD1 inhibitor developed by our group as a specific probe. ZY0511 profoundly modified the human colorectal and
\end{abstract}

Correspondence to: Professor Yinglan Zhao, State Key Laboratory of Biotherapy and Cancer Center, West China Hospital, West China Medical School, and Collaborative Innovation Center for Biotherapy, Sichuan University, No. 17, 3rd Section, Ren Min South Road, Chengdu, Sichuan 610041, P.R. China

E-mail: zhaoyinglan@scu.edu.cn

*Contributed equally

Abbreviations: LSD1, lysine specific demethylase 1; AML, acute myeloid leukemia; S1P, sphingosine-1-phosphate; GCS, glucosylceramide synthase; CERK, ceramide kinase; AC, acid ceramidase; SPHK, sphingosine kinase; UPLC/Q-TOF-MS, ultra-performance liquid chromatography-quadrupole time of flight mass spectrometry; PCA, principal component analysis; OPLS-DA, supervised orthogonal partial least squares discriminant analysis; VIP, variable importance; GL, glycerophospholipid; SP, sphingolipid; PS, phosphatidylserine; PC, phosphatidylcholine; PE, phosphatidylethanolamine; PI, phosphatidylinositol; PA, phosphatidic acid; PG, phosphatidylglycerol; Cer, ceramide; GluCer, glucosylceramide; LacCer, lactosylceramide; CerP, ceramide phosphate; SM, sphingomyelin; SPTL, serine palmitoyltransferase; CERS, ceramide synthase; DEGS, dihydroceramide desaturases; SGPP1, sphingosine-1-phosphate phosphatase 1; SGPL1, sphingosine-1-phosphate lyase 1

Key words: LSD1, LSD1 inhibitor, lipidomics, sphingolipids, ceramide cervical cancer cell lipid metabolism. A total of 256 differential metabolites were identified in HeLa cells, and 218 differential metabolites were identified in HCT116 cells, respectively. Among these lipid metabolites, phosphatidylserine, phosphatidylethanolamine, phosphatidylcholine and sphingomyelin (SM) were downregulated by ZY0511. In contrast, ceramide (Cer) and a small portion of glycerophospholipids such as phosphatidylinositol and phosphatidylethanolamine were upregulated by ZY0511. These results revealed a disturbance in sphingolipids (SPs) and glycerophospholipids, which may be correlated with the progression of cancer. Furthermore, a marked increase in Cer and prominent decrease in SM were consistent with the upregulated expression of key enzymes in the Cer synthesis process including de novo synthesis, hydrolysis of SM and the salvage pathway after ZY0511 exposure. In conclusion, our research reveals a link between LSD1 and lipid metabolism in cancer cells, offering more comprehensive evidence for the application of LSD1 inhibitors for cancer therapy. The underlying mechanisms of how the LSD1 inhibitor regulates lipid metabolism warrant further investigation.

\section{Introduction}

Dysregulated epigenetic processes play a critical role in cancer onset and progression. In contrast to DNA mutations, epigenetic modifications are reversible, thus suitable for pharmacological interventions. Lysine-specific demethylase 1 (LSD1), also known as KDM1A or AOF2, specifically remove the methyl group from mono and demethylated histone $\mathrm{H} 3$ at lysine 4 (1) or lysine 9 (2). Overexpression of LSD1 is observed in many types of cancer including prostate cancer $(3,4)$, ovarian cancer (5), breast cancer (6), esophageal squamous cancer (7), colorectal cancer (8), acute myeloid leukemia (9) and small cell lung cancer (10) and is associated with the poor survival rate of cancer patients. Machinery by which LSD1 regulates cancer progression has been widely investigated. LSD1 was found to sustain the leukemogenic potential of MLL-AF9 leukemia stem cells (11). LSD1 was also found to inhibit the expression of myeloid differentiation-associated 
genes that decreased the differentiation of leukemia cells, and LSD1 inhibition reactivated the ATRA (all-trans-retinoic acid) differentiation pathway in acute myeloid leukemia (AML), thus sensitizing AML cells to ATRA (9). In addition to leukemia, a number of studies have demonstrated the important role of LSD1 in solid tumors. LSD1 specifically interacts with the androgen receptor (2), the estrogen receptor (12) or large chromatin-modifying corepressor complexes $(13,14)$, and regulates prostate cancer (15). Although these studies reveal roles for LSD1 in solid tumors, the underlying epigenetic mechanism is still poorly understood.

Cancer cells undergo extensive metabolic reprogramming to sustain tumor growth. In addition to well-known glycolysis and glutamine metabolism, lipid metabolism has attracted great interest in tumorigenesis. Lipids form a diverse group of water-insoluble biomolecules that include triacylglycerides, glycerophospholipids, SPs, sterols and others. There is increasing evidence that lipids play critical roles in tumorigenesis and progression, especially as second messengers or hormones in signaling (16). Changes in lipid metabolism affect many cancer cell processes, including cell growth, proliferation, motility, autophagy (17) and apoptosis (18). Therefore, the study of lipid metabolism is of great significance to elucidate the pathogenesis of tumors and to discover effective therapeutic targets for tumors. Among the different types of lipids, SPs regulate various biological processes by controlling the signaling functions of cancer cell signal transduction network $(19,20)$. Cer and sphingosine are two bioactive SPs which are produced through three main pathways, including the de novo synthesis pathway, SM hydrolysis and the salvage pathway under cellular stress $(19,21)$. Their decreases are associated with tumorigenesis and progression. Under the treatment of chemotherapy, radiation and oxidative stress, the levels of Cer and sphingosine are induced which then mediate cell death, senescence and cell cycle arrest (22). In contrast, high levels of sphingosine-1-phosphate (S1P), SM and glucosylceramide which are members of the SPs possess anti-apoptotic roles $(23,24)$. Although the above-mentioned studies indicate the critical characters of SPs in cancer cells, lipid metabolic contributions to cancer cell epigenetic alterations are largely unknown. LSD1 is implicated in cancer cell metabolism and may regulate glycolytic and mitochondrial metabolism in hepatocellular carcinoma (HCC) cells (25) and esophageal cancer (26). However, few studies have investigated the impact of LSD1 in the lipidomic profiling of cancer cells, and the lipidome remodeling of cancer cells regulated by LSD1 remain largely elusive.

Lipidomics was recently developed to study the total lipid composition of a body. With more than 180,000 different molecular species, lipids form a vast class of biomolecules, and all of these lipids possess a unique chemical structure and biological activities. Recent advances in technologies such as spectroscopy, chromatography and most importantly in MS (mass spectrometry) make lipidomics a mature field such as genomics, transcriptomics, proteomics and metabolomics. Recently, the application of LC/MS-MS (liquid chromatography-tandem mass spectrometry) facilitates the precise profiling of lipid species even including those low abundance lipid classes (27). The analysis of lipid profiling in cancer cells provides an unbiased method by which to fully understand the mechanism of cancer inhibitors, meanwhile providing a benefit to lipid biomarker development.

In the present study, we performed an ultra-performance liquid chromatography (UPLC) ESI-Q-TOF-MS (electrospray ionization quadrupole time of flight mass spectrometry)-based lipidomics approach to investigate the LSD1 medicated lipid profiling in human cancer cells. ZY0511, a novel, potent selective LSD1 inhibitor with a half maximal inhibitory concentration $\left(\mathrm{IC}_{50}\right)$ value of $1.7 \mathrm{nM}$, which was previously developed by our group, was used as a specific LSD1 probe (28). Our research indicated that LSD1 inhibitor ZY0511 modified the lipidome of cancer cells especially the level of sphingolipids Cer and SM which indicates a critical role of LSD1 in lipid metabolism. These findings promote a better understanding of the correlation between LSD1 and lipid metabolism in cancer.

\section{Materials and methods}

Preparation and enzyme activity detection of ZY0511. ZY0511 [chemical name: (E)-N'-(2,3-dihydro-1H-inden-1-ylidene) benzohydrazides] was synthesized at the State Key Laboratory of Biotherapy, Sichuan University (Sichuan, China) and was purified to $>99 \%$ purity as determined by high performance liquid chromatography (HPLC). Its structural formula is shown in a previous study (28). For in vitro assays, ZY0511 was dissolved in DMSO for which the final concentration was no more than $0.1 \%(\mathrm{v} / \mathrm{v})$ after addition to the cells. The detection of enzyme activity of ZY0511 was also performed as described in a previous study (28).

Cell culture and reagents. HeLa (human cervical cancer cell line) and HCT116 (human colorectal cancer cell line) were purchased from the American Type Culture Collection (ATCC). Cells were maintained in RPMI-1640 media or DMEM media (Gibco; Thermo Fisher Scientific, Inc.) supplemented with $10 \%$ fetal bovine serum (FBS) (Hyclone; GE Healthcare), $100 \mathrm{U} \mathrm{ml}^{-1}$ penicillin (Sigma-Aldrich; Merck $\mathrm{KGaA}$ ) and $100 \mu \mathrm{g} \mathrm{ml}^{-1}$ streptomycin (Sigma-Aldrich; Merck $\mathrm{KGaA})$ at $37^{\circ} \mathrm{C}$ in a humidified atmosphere containing $5 \% \mathrm{CO}_{2}$. All cancer cell lines were maintained according to ATCC recommended procedures.

Sample preparation and extraction. HeLa and HCT116 cells were treated with $2 \mu \mathrm{M}$ ZY0511 for $48 \mathrm{~h}$. Sample extraction process was according to the Folch method (29). Briefly, cells were collected and washed with cold PBS, then $300 \mu \mathrm{l}$ chloroform was added, followed by being vortexed for $10 \mathrm{sec}$. Then, $150 \mu 1$ methyl alcohol was added, and the samples were vortexed for $15-20 \mathrm{~min}$ at $4^{\circ} \mathrm{C}$. Finally, $135 \mu 10.9 \% \mathrm{NaCl}$ solution was added, followed by centrifugation for $1,500 \mathrm{x} \mathrm{g}$ for $3 \mathrm{~min}$. This procedure separated the suspension into three phases: A water phase at the top, a denatured protein phase in the middle, and a lipid phase at the bottom. The lipid phase of each sample was collected and evaporated to dryness under a stream of nitrogen, and then frozen at $-80^{\circ} \mathrm{C}$ for further study.

$U P L C / Q-T O F-M S$. Individual MS was run for 'each' cell homogenate in the study. Each experiment was repeated 6 times independently and the samples were not pooled. The dried lipid samples were reconstituted with acetonitrile:isopropanol 
(7:3, v/v) solution (300 $\mu 1$ per mg of lipids), followed by ultrasonicated and centrifuged at $14,000 \mathrm{x}$ g for $10 \mathrm{~min}$. The $100 \mu \mathrm{l}$ supernatant was transferred to insert pipes (Agilent Technologies, Inc.), and then separated by an ACQUITY UPLC (Waters Ltd.) and analyzed by ESI-Q-TOF-MS (Waters Ltd.). The injection volume was $3 \mu \mathrm{l}$, and the column which was used for separation was ACQUITY UPLC HSS T3 column (1.8 $\mu \mathrm{m}, 2.1 \times 100 \mathrm{~mm}$; Waters Ltd.) and the work temperature was $55^{\circ} \mathrm{C}$. The mobile phase A was acetonitrile:water $(4: 6, \mathrm{v} / \mathrm{v})$ with $5 \mathrm{mM}$ ammonium acetate added. The mobile phase B was isopropanol:water $(9: 1, \mathrm{v} / \mathrm{v})$ with $5 \mathrm{mM}$ ammonium acetate added. The flow rate of the mobile phase was $0.4 \mathrm{ml} / \mathrm{min}$. A linear gradient was used as follows: $40-70 \% \mathrm{~B}$ at $0-3 \mathrm{~min}$, $70-95 \% \mathrm{~B}$ at 3-14 $\mathrm{min}, 95 \% \mathrm{~B}$ at $14-15.5 \mathrm{~min}$. The column was equilibrated for $3.5 \mathrm{~min}$ before injection, giving a total run time of $20 \mathrm{~min}$.

At the end of the ultra-high liquid phase separation, the MS was operated in the positive and negative ionization modes, respectively. The capillary voltage was $2.5 \mathrm{kV}$ and the sample cone voltage was $30 \mathrm{~V}$ in positive ionization modes. The capillary voltage was $2.5 \mathrm{kV}$ and the sample cone voltage was $-25 \mathrm{~V}$ in negative ionization modes. The source temperature was $120^{\circ} \mathrm{C}$, the desolation gas flow was 800 liters $/ \mathrm{h}$, and the cone gas flow was 20 liters $/ \mathrm{h}$. The mass of 50-1,200 (m/z) was acquisition under altering full scan and all ion fragmentation scan mode. A lock spray was required for analyses to ensure accuracy. Under the positive ion mode, the accurate $[\mathrm{M}+\mathrm{H}]^{+}$was 556.2771, while the negative ion mode, the accurate $[\mathrm{M}+\mathrm{H}]^{-}$was 554.2615 . The data were collected in the continuous mode using MassLynx (version 1.0; Waters Ltd.).

Data processing. The original data collected from MS were imported with Progenesis QI software (version 2.0; Waters) to align, match and correct peaks. Then peaks were picked and lipids metabolites were identified by referring to the Human Metabolome Database (www.hmdb.ca) and the Lipid Maps Database (www.lipidmaps.org). The score of mass error, fragment and isotope similarity help identify the lipids in the Progenesis QI software. Then data sheets from the Progenesis QI software were obtained and absolute intensities of all identified compounds were recalculated to the relative abundances of the lipid molecules. After that, the data were exported to EZinfo software (version 2.0; Umetrics), and data analysis was performed to obtain group clusters such as unsupervised principal components analysis (PCA) and supervised orthogonal partial least squares discriminant analysis (OPLS-DA). The variable importance (VIP >1) was obtained from EZinfo software and $\mathrm{P}<0.05$ (Student's t-test) of each identified metabolite was obtained from Progenesis QI to estimate the significance of the changes in the metabolites between the control and treated group. Volcano plot and pathway analysis were performed by MetaboAnalyst (http://www.metaboanalyst.ca) which is an online metabolomic analysis software.

Real-time quantitative PCR. RNA was extracted using Total Miniprep kit (Axygen). cDNA was synthesized using SuperScript IV Reverse Transcriptase (Thermo Fisher Scientific, Inc.). The amount of cDNA was then quantified by the Bio-Rad CFX Real-Time PCR detection system using SYBR Green SuperMix (Bio-Rad, Inc.) and the primer sequences are listed in Table I. All samples were tested in triplicate and expression levels were normalized to $\beta$-actin mRNA. The reaction process was as follows: Initial denaturation: $95^{\circ} \mathrm{C}, 30 \mathrm{sec}$; denaturation: $95^{\circ} \mathrm{C}, 5 \mathrm{sec}$; annealing and elongation: $60^{\circ} \mathrm{C}, 20 \mathrm{sec}$; cycles: 40 . The expression was calculated with the comparative method $\left(2^{-\Delta \Delta \mathrm{Cq}}\right)(30)$.

Gene correlation analysis. UCSC Xena (http://xena.ucsc.edu/) is an open database which is used to explore and download The Cancer Genome Atlas (TCGA datasets) (31). The gene expression RNAseq data (IlluminaHiSeq) of all cervical squamous cell carcinoma and endocervical adenocarcinoma (CESC) and colon adenocarcinoma (COAD) clinical samples collected by TCGA were downloaded from UCSC Xena. Then gene correlation analysis including Pearson analysis and statistical analysis of all clinical samples was carried out by GraphPad Prism 8.0.1 (GraphPad Software, Inc.).

Statistical analysis. All data are represented as means \pm SD as indicated in the figure legends. Comparisons between two groups such as the control group and the treated group were analyzed using unpaired, two tailed t-test. P-value $<0.05$ was considered indicative of statistical significance. The Pearson's correlation test was used to investigate the correlation between gene expression. GraphPad Prism 8.0.1 was used for statistical analysis.

\section{Results}

Orthogonal projections to latent structures discriminant analysis (OPLS-DA) model. We previously found that ZY0511 possessed strong LSD1 inhibition activity, and markedly inhibited human cancer cell growth $(28,32)$. In previous research, we explored the anti-proliferative activity of ZY0511 for long-term treatment such as 72 and $96 \mathrm{~h}$ and the growth of cancer cells was inhibited. Thus, we collected the lipids of cancer cells for the $48 \mathrm{~h}$ treatment and ZY0511 was used as a specific probe to explore the role of LSD1 in cancer lipid metabolism. The OPLS-DA model was performed to analyze the metabolite differences after ZY0511 treatment. The results showed a clear differentiation between the control groups and the ZY0511-treated groups in the two cell lines (Fig. 1). Both the $\mathrm{Q}^{2}$ and $\mathrm{R}^{2}$ values of the two cell line models were $>0.5$ in the positive and negative ion modes [(A) HeLa pos and (B) HeLa neg; (D) HCT116 pos and (E) HCT116 neg] which indicated the good prediction abilities of these two models.

Meanwhile, the original data were transported to online MetaboAnalyst software to obtain volcano spots of these two cell line models. As shown in Fig. 1C and F, important features were selected by volcano spots with fold change $>2$ and $\mathrm{P}<0.05$ (the dotted lines in Fig. 1C and F represent the thresholds). All of these results indicate that the ZY0511 treatment significantly modified the lipid profile in these cancer cell lines.

ZY0511 treatment modifies the lipidome of cancer cells. Lipidomic profiles in the HeLa and HCT116 cells were significantly altered by ZY0511 treatment for $48 \mathrm{~h}$. The distinguished lipid metabolites in these two cell line models, which satisfied VIP $>1$ and $\mathrm{P}<0.05$, are summarized by class (Figs. 2-4). The top metabolites with a fold-change in the cancer cells are 
Table I. Primer sequences of qPCR.

\begin{tabular}{|c|c|c|}
\hline Name & Forward & Reverse \\
\hline SPTLC1 & CAGTGCTATTCCTGCTTACTCT & TTCTTTTAGTAGTCGCTCGAGG \\
\hline SPTLC3 & CGTGGATTATTTACGGGTTCAC & CAGAGGAACAACAGAAGCATTC \\
\hline CERS2 & GGAGTCAGCCAAGATGTTTAAC & CCAGTCGGGTGATGATAAAAAC \\
\hline CERS4 & CAGTTTCAACGAGTGGTTTTGG & CAGGCCAATGAATCTCTCAAAG \\
\hline CERS5 & CTTCGAGCGATTTATTGCCAAA & CTCCAGCCTTTTCTTATCAGGA \\
\hline CERS6 & AAGACGCAATCAGGAGAAGCCAAG & AGCAATGCCTCGTATTCCACAACC \\
\hline$D E G S 1$ & GGAGCTGATGGCGTCGATGTAG & AAGTGACCTGTGCCACGGTATTG \\
\hline$D E G S 2$ & CAGCCCTTCTTCTACTCACTAC & ATAGTAGGAGTAGGTCTCGTGG \\
\hline SGPL1 & CGTGATTTTGACATCTACCGAC & TGGTCTTCGCTTTAGGATTCTT \\
\hline$S G P P 1$ & CTGGTGTTCTCTAGTTTGCCTA & GTGAGTTTGGTTGAAGTTGTCA \\
\hline SMPD1 & ATCTGGAAGGCAAAGGTGTG & CAGAGGCAGAGCAGAGGAAC \\
\hline$S M P D 2$ & CAAGGTGAGGACTTGCCTGT & ACCATTGTGTTGCCTTCCTC \\
\hline SMPD3 & AGTCACCCAAGCCACATTTC & GCAGCAACTGTCCAACAGAA \\
\hline SMPD4 & TTCGCTTGAGTCTGGGAGTT & CCCAACTAGCGGGAACTACA \\
\hline ENPP7 & CTCAGGATATGCAGCGAACA & СТTCTTTGCCATCACAAGCA \\
\hline$\beta$-actin & CATGTACGTTGCTATCCAGGC & СТCCTTAATGTCACGCACGAT \\
\hline
\end{tabular}

SPTL, serine palmitoyltransferase; CERS, ceramide synthase; DEGS, dihydroceramide desaturases; SGPP1, sphingosine-1-phosphate phosphatase 1; SGPL1, sphingosine-1-phosphate lyase 1; SMPD, sphingomyelin phosphodiesterase; ENPP7, ectonucleotide pyrophosphatase/phosphodiesterase family member 7 .
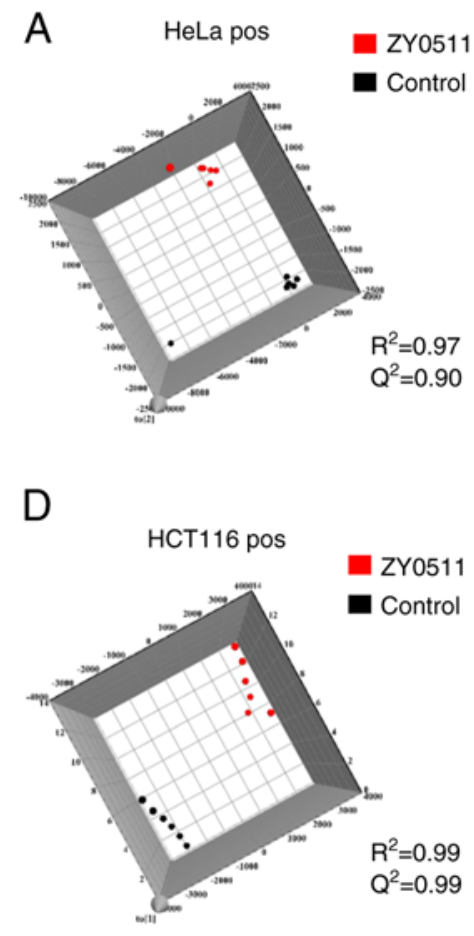

B

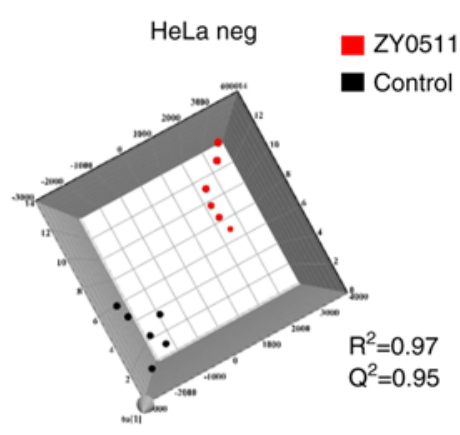

E

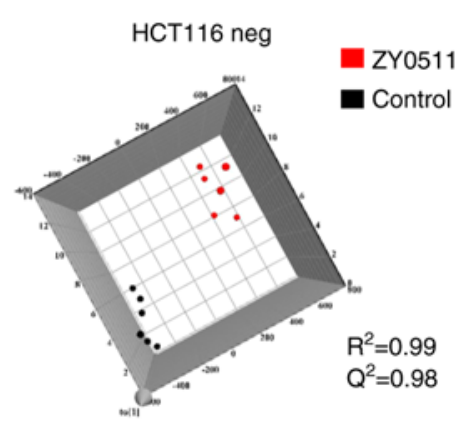

C

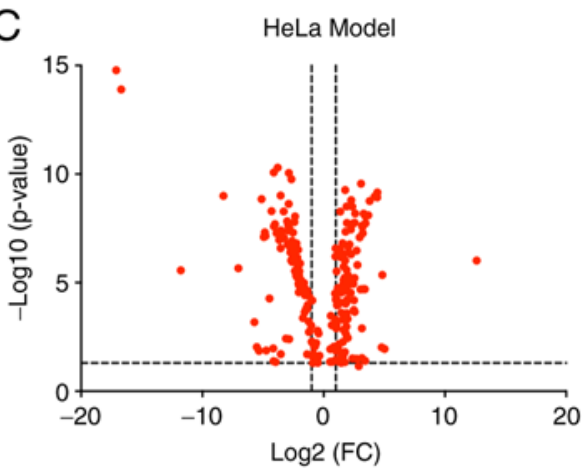

$\mathrm{F}$

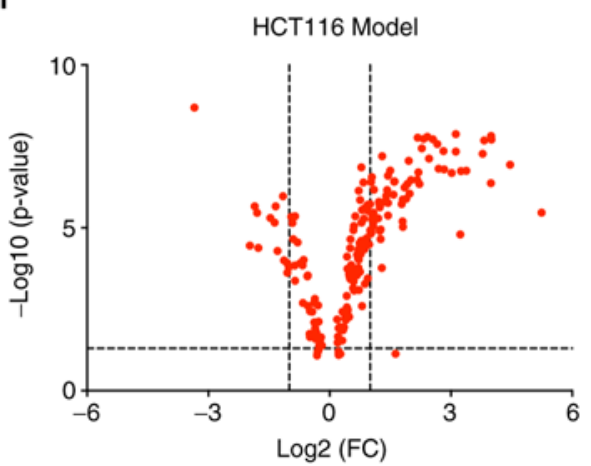

Figure 1. Metabolite profile difference between the control and ZY0511-treated group. (A and B) Metabolite profile difference in the HeLa cell line treated with ZY0511 in positive (pos) (A) and negative (neg) (B) ion modes. (C) Important features selected by a volcano plot with fold-change threshold $>2$ and t-test threshold $<0.05$ in the HeLa model; the further its position away from the point $(0,0)$, the more significant is the feature. (D and E) Metabolite profile difference in the HCT116 cell line treated with ZY0511 in positive (pos) (D) and negative (neg) (E) ion modes. (F) Important features selected by a volcano plot with fold-change threshold $>2$ and $t$-test threshold $<0.05$ in the HCT116 model; the further its position away from the point $(0,0)$, the more significant is the feature.

shown in Tables II and III. As glycerophospholipids (GLs) and SPs are the main types of lipids in cells and are correlated with many biological processes, we primarily focused on the change in these two types of lipid metabolites. 
A

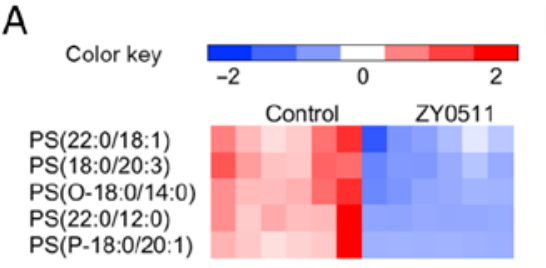

D

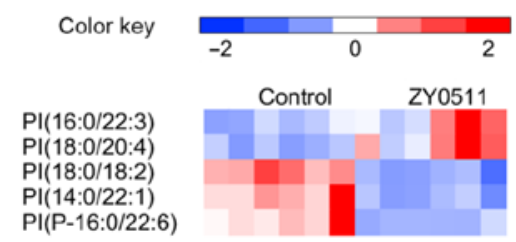

E

Color key

PG(16:0/18:1) PG(22:4/0:0)
B

Color key

PC(O-16:0/18:2)

$\mathrm{PC}(24: 0 / 18: 0)$

$\mathrm{PC}(20: 0 / 20: 2)$

$\mathrm{PC}(18: 4 / 16: 1)$

$\mathrm{PC}(16: 1 / 14: 0)$

PC(16:1/16:1)

$\mathrm{PC}(16: 1 / 16: 0)$

$\mathrm{PC}(22: 0 / 16: 0)$

PC(18:1/16:1)

$\mathrm{PC}(16: 1 / 18: 3)$

$\mathrm{PC}(20: 5 / 18: 3)$

PC(P-18:1/14:0)

PC(O-20:1/20:4)

$\mathrm{PC}(18: 0 / 14: 0)$

$\mathrm{PC}(18: 1 / 16: 0)$

$\mathrm{PC}(18: 1 / 18: 3)$

$\mathrm{PC}(20: 1 / 18: 4)$

$\mathrm{PC}(18: 1 / 18: 1)$

$\mathrm{PC}(\mathrm{O}-24: 0 / 20: 4)$

$\mathrm{F}$

Color key

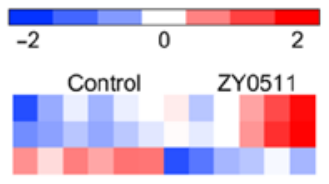

PA(16:1/18:1) $\mathrm{PA}(\mathrm{O}-20: 0 / 22: 0)$ $\mathrm{PA}(20: 3 / 18: 0)$ PA(20:1/22:6) PA(18:0/16:0) $\mathrm{PA}(\mathrm{O}-20$ :0/16:0) $\mathrm{PA}(\mathrm{P}-16: 0 / 22: 2)$

PA(22:0/22:0)

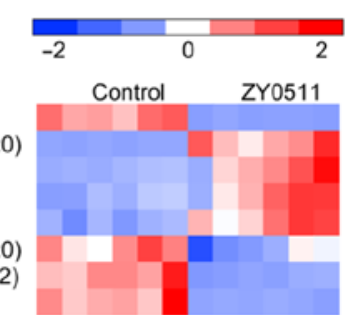

C

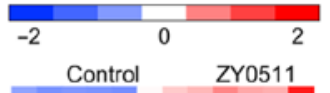

Color key

PE(18:3/16:0)

PE(14:0/18:1)

PE(16:0/18:2)

PE(18:2/14:0)

PE(14:0/20:1)

PE(14:1/22:2)

PE(O-20:0/12:0)

PE(P-18:1/16:0)

PE(14:0/P-18:1)

PE(20:3/16:1)

PE(20:1/20:1)

PE(18:0/18:0)

PE(20:1/20:3)

PE-NMe(16:0/18:1)

$\mathrm{PE}(16: 0 / 20: 4)$

PE(14:0/20:0)

PE(16:0/20:3)

PE(18:1/18:1)

PE(18:0/20:4)

PE(18:1/16:0)

$\mathrm{PE}(18: 1 / 20: 4)$

PE(20:1/18:1)

PE(18:0/18:1)

PE(18:0/18:2)

PE(20:0/18:3)

PE(24:1/14:1)

$\operatorname{PE}(20: 2 / 20: 1)$

PE(P-18:1/20:3)

PE(22:1/18:0)

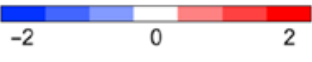

Control ZY0511

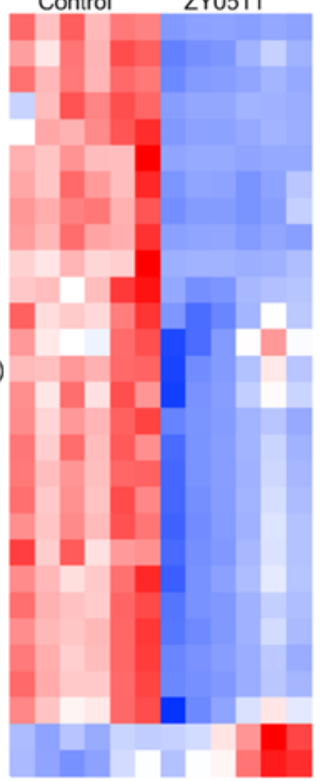

Figure 2. Comparative GL profile expression in the HeLa model. Heatmap display of unsupervised hierarchical clustering of altered lipids in GLs including (A) PS, (B) PC, (C) PE, (D) PI, (E) PG and (F) PA (n=6). Data are normalized by the mean value of each group. VIP $>1$ and P $<0.05$ are required for all displayed features. Statistical analysis for individual lipid species data is based on the unpaired two-tailed Student t-test. GL, glycerophospholipid; PS, phosphatidylserine; PC, phosphatidylcholine; PE, phosphatidylethanolamine; PI, phosphatidylinositol; PG, phosphatidylglycerol; PA, phosphatidic acid.

A

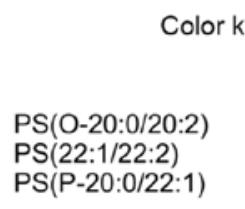

C

$P G(18: 0 / 18: 2)$
$P G(20: 3 / 22: 0)$

PG(P-20:0/20:1)

D

PE(14:0/18:0)
PE(P-20:0/22:4)
PE(18:0/18:3)
PE(22:4/24:1)
PE(O-18:0/18:2)
PE(20:1/24:0)
PE(22:2/24:1)
PE(24:0/22:4)
PE(24:1/18:1)
PE-NMe(O-16:0/O-16:0)
PE(O-16:0/O-16:0)
PE(22:1/P-18:1)
PE-NMe2(18:1/18:1)
PE-NMe2(O-16:0/O-16:0)
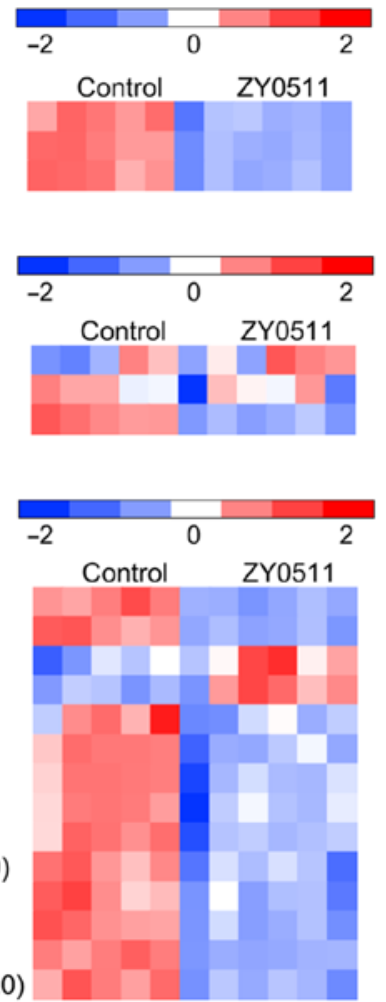

B

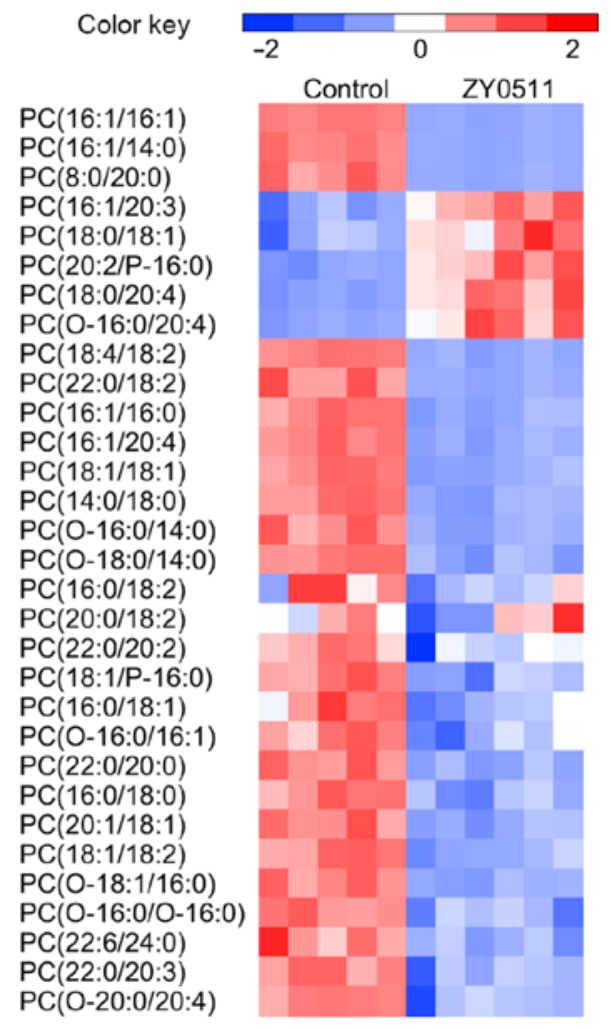

Figure 3. Comparative GL profile expressing in the HCT116 model. Heatmap display of unsupervised hierarchical clustering of altered lipids in GLs including (A) PS, (B) PC, (C) PG and (D) PE (control group: $n=5$; treated group: $n=6$ ). Data are normalized by the mean value of each group. VIP $>1$ and P<0.05 are required for all displayed features. Statistical analysis for individual lipid species data based on the unpaired two-tailed Student t-test. GL, glycerophospholipid; PS, phosphatidylserine; PC, phosphatidylcholine; PG, phosphatidylglycerol; PE, phosphatidylethanolamine. 
A

Color key
Cer(d18:0/24:1)
HexoCer (d18:1/26:1)
Etn-1-P-Cer(d18:0/14:1)
CerP(d18:1/18:0)
CerP(d18:1/24:1)
GluCer(d18:1/18:0)
Cer(d18:1/24:1)
GluCer(d18:1/24:1)
GluCer(d18:1/16:0)
LacCer (d18:1/24:1)

C
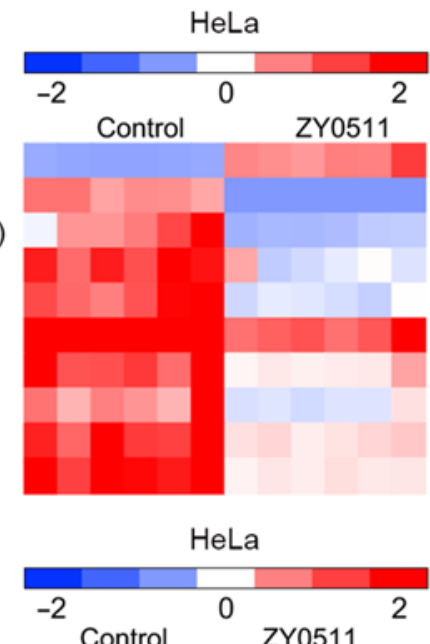

CerP(d18:1/22:0)

Cer(d18:0/24:1)

GlcCer(d18:1/20:0)

LacCer(d18:0/24:1)

GalCer(d18:1/16:0)

LacCer(d18:1/20:0)

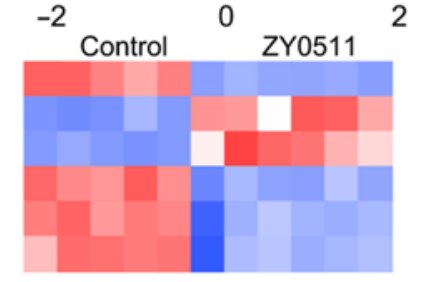

B

Color key

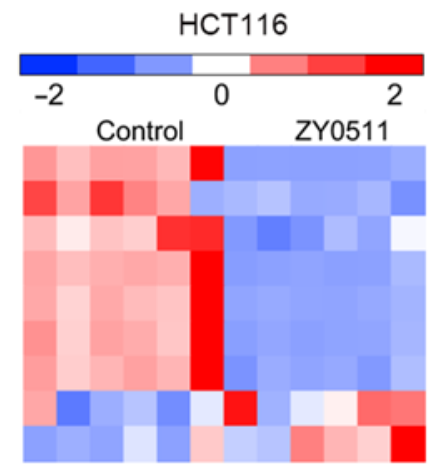

D

Color key

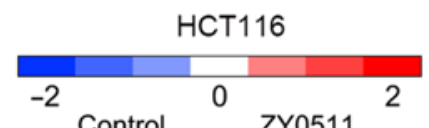

$\mathrm{SM}(\mathrm{d} 18: 1 / 16: 0)$

SM(d18:0/24:1)

SM(d18:1/14:0)

$\operatorname{SM}(d 18: 1 / 16: 1)$

$\mathrm{SM}(\mathrm{d} 18: 0 / 16: 1)$

SM(d18:1/24:1)

SM(d18:1/18:0)

Figure 4. Comparative SP profile expression in the HeLa and HCT116 models. Heatmap display of unsupervised hierarchical clustering of altered lipids in SPs including (A) Cer, CerP, GluCer and LacCer in the HeLa model (n=6), (B) SM in the HeLa model (n=6), (C) Cer, CerP, GluCer and LacCer in the HCT116 model (control group: $n=5$; treated group: $n=6$ ), and (D) SM in the HCT116 model (control group: $n=5$; treated group: $n=6$ ). Data are normalized by the mean value of each group. VIP $>1$ and $\mathrm{P}<0.05$ are required for all displayed features. Statistical analysis for individual lipid species data are based on the unpaired two-tailed Student t-test. SP, sphingolipid; Cer, ceramide; CerP, ceramide phosphate; GluCer, glucosylceramide; LacCer, lactosylceramide; SM, sphingomyelin.

Compared with the control group, ZY0511 treatment resulted in marked alterations of GLs and SPs. In HeLa cells, most of the GLs were decreased after ZY0511 treatment (Fig. 2A-C) including phosphatidylserine (PS), phosphatidylcholine (PC) and phosphatidylethanolamine (PE). PS upregulation in cancer cells can inhibit the immune response and promote tumorigenesis (33), which suggest PS downregulation could benefit tumor inhibition. In addition, the altered levels of phosphatidylinositol (PI), phosphatidic acid (PA) and phosphatidylglycerol (PG) were undetermined (Fig. 2D-F). The levels of long-chain PIs such as PI (16:0/22:3) and PI (18:0/20:4) were increased by ZY0511, while the levels of short-chain PIs such as PI (18:0/18:2) and PI (14:0/22:1) were decreased (Fig. 2D). In addition, the expression of PA and PG changed randomly independent of the length of chains and saturability of the lipids. In the HCT116 models, we found that most of the PSs (Fig. 3A), PGs (Fig. 3C) and PEs (Fig. 3D) were decreased by ZY0511 treatment, only a few of the PCs such as PC (16:1/20:3), PC (18:0/18:1) and PC (18:0/24:4) were increased by ZY0511 treatment (Fig. 3B). Inhibition of PC phospholipase activity was found to decrease ovarian cancer cell proliferation (34), which suggest that the increase of PC expression is closely related to the occurrence of tumors. As cancer cells are highly proliferating and need plenty of GLs for its membrane production, the decrease in GLs after ZY0511 treatment may be an important reason for the anti-proliferative effect of ZY0511 against cancer cells (35).

ZY0511 treatment resulted in marked alterations in SP metabolism. A significant increase in anti-survival metabolites Cer and marked decrease in pro-survival metabolites glucosylceramide (GluCer) and lactosylceramide (LacCer) were observed in the two cell lines after ZY0511 treatment (Fig. 4A and C). The cellular level of SM was decreased by ZY0511 (Fig. 4B and D). These results suggested that the Cer synthesis in the cancer cells was activated following ZY0511 treatment. The hydrolysis from SM to Cer may also be promoted by ZY0511 exposure. A high level of Cer was found to lead to an obvious increase in apoptosis in cancer such as in leukemic (36) and HeLa cells (37). The roles of Cer biogenesis were later revealed which are required for BAX integration in HeLa cells (37). LSD1 plays an important role in the process of apoptosis (9), the association between LSD1 and apoptosis may be due to the induction of Cer in cells after LSD1 inhibition.

In conclusion, cellular lipid metabolism was obviously dysregulated by ZY0511. Most of the GLs including PC, PS and $\mathrm{PE}$ were downregulated in the two cell line models. More importantly, the levels of bioactive metabolites of SP metabolism such as Cer and SM were influenced to a great degree which may be a possible anticancer mechanism of ZY0511.

The lipid metabolism especially SP metabolism is significantly modified by ZY0511. Next, we explored which pathway was markedly modified by the treatment of ZY0511. We performed pathway enrichment analysis in MetaboAnalyst. The results showed the lipid metabolism pathways which were altered by ZY0511 including SP metabolism, GL metabolism, arachidonic acid metabolism and others. Among these pathways, SP metabolism was the most dysregulated pathway under the inhibition of LSD1 by ZY0511 in both cell line models (Fig. 5A and B). In HeLa cells, four metabolites including LacCer (d18:1/24:1(15Z)), Cer (d18:0/24:1), GluCer (d18:1/16:0) 
Table II. The top metabolites with fold-change in the HeLa cell model following ZY0511 treatment.

\begin{tabular}{|c|c|c|c|c|c|c|c|c|}
\hline Compound ID & Compound & P-value & $\log 10 p$ & Score & $\begin{array}{l}\text { Isotope } \\
\text { similarity }\end{array}$ & $\mathrm{m} / \mathrm{z}$ & $\begin{array}{l}\text { Retention } \\
\text { time (min) }\end{array}$ & $\begin{array}{l}\log 2 \\
(\mathrm{FC})\end{array}$ \\
\hline HMDB02421 & 7-Sulfocholic acid & $1.64 \mathrm{E}-15$ & 14.79 & 55.60 & 87.14 & 487.24 & 3.88 & 17.11 \\
\hline LMFA08020108 & $\mathrm{N}$-oleoyl threonine & $2.72 \mathrm{E}-06$ & 5.57 & 39.80 & 77.53 & 767.62 & 8.45 & 11.78 \\
\hline LMGP04050033 & PG(17:0/0:0) & $2.18 \mathrm{E}-06$ & 5.66 & 41.10 & 91.83 & 543.29 & 2.27 & 7.03 \\
\hline HMDB11767 & $\operatorname{Cer}(\mathrm{d} 18: 0 / 23: 0)$ & $6.56 \mathrm{E}-04$ & 3.18 & 40.10 & 64.14 & 682.63 & 7.97 & 5.72 \\
\hline HMDB04957 & Cer (d18:1/25:0) & $9.29 \mathrm{E}-03$ & 2.03 & 43.90 & 88.51 & 708.65 & 7.96 & 5.52 \\
\hline LMGL02010311 & $\operatorname{DG}(16: 0 / 0: 0 / 16: 0)(\mathrm{d} 5)$ & $1.42 \mathrm{E}-09$ & 8.85 & 38.90 & 95.94 & 591.57 & 12.76 & 5.12 \\
\hline HMDB11146 & DG(16:0e/18:0/0:0) & 7.94E-08 & 7.10 & 42.40 & 95.25 & 605.55 & 12.02 & 4.94 \\
\hline LMGP10020071 & $\mathrm{PA}(\mathrm{O}-20: 0 / 22: 0)$ & 4.88E-08 & 7.31 & 36.50 & 82.39 & 773.65 & 7.20 & 4.86 \\
\hline HMDB07166 & DG(18:0/20:1(11Z)/0:0) & 7.14E-08 & 7.15 & 52.80 & 95.97 & 633.58 & 12.51 & 4.80 \\
\hline LMGP04050008 & $\mathrm{PG}(16: 0 / 0: 0)$ & $1.34 \mathrm{E}-02$ & 1.87 & 46.70 & 93.26 & 507.27 & 2.08 & 4.74 \\
\hline LMFA08020128 & $\mathrm{N}$-oleoyl glutamine & 5.33E-05 & 4.27 & 41.90 & 97.97 & 409.31 & 4.49 & 4.47 \\
\hline LMGL03011253 & $\begin{array}{l}\mathrm{TG}(18: 0 / 20: 3(8 \mathrm{Z}, 11 \mathrm{Z}, 14 \mathrm{Z}) / \\
21: 0)[\text { iso6] }\end{array}$ & $5.08 \mathrm{E}-09$ & 8.29 & 36.10 & 72.93 & 977.85 & 12.12 & 4.30 \\
\hline HMDB11636 & Salicyl CoA & $1.06 \mathrm{E}-02$ & 1.97 & 35.90 & 85.83 & 903.20 & 11.55 & 4.15 \\
\hline LMGP10010598 & $\mathrm{PA}(20: 3(8 \mathrm{Z}, 11 \mathrm{Z}, 14 \mathrm{Z}) / 18: 0)$ & $4.13 \mathrm{E}-02$ & 1.38 & 37.20 & 88.84 & 771.51 & 4.12 & 4.13 \\
\hline HMDB07156 & DG(18:0/16:0/0:0) & 2.57E-08 & 7.59 & 40.60 & 99.36 & 619.53 & 7.07 & 4.11 \\
\hline HMDB07187 & DG(18:1(11Z)/18:0/0:0) & $2.01 \mathrm{E}-08$ & 7.70 & 43.10 & 97.28 & 605.55 & 12.02 & 4.02 \\
\hline LMFA01020381 & $\begin{array}{l}\text { 17:4(2E,4E,9E,11E) } \\
(8 \mathrm{Me}[\mathrm{R}], 10 \mathrm{Me}, 15 \mathrm{Me}[\mathrm{R}])\end{array}$ & $4.50 \mathrm{E}-02$ & 1.35 & 58.60 & 99.76 & 303.23 & 2.34 & 3.96 \\
\hline HMDB44730 & TG(18:0/16:0/20:1(11Z)) & $5.20 \mathrm{E}-08$ & 7.28 & 49.70 & 96.38 & 906.85 & 12.49 & 3.88 \\
\hline HMDB11769 & Cer(d18:0/24:1(15Z)) & $5.09 \mathrm{E}-11$ & 10.29 & 40.00 & 95.73 & 650.65 & 7.43 & 3.79 \\
\hline HMDB43920 & TG(16:0/18:0/18:1(11Z)) & $1.09 \mathrm{E}-07$ & 6.96 & 53.40 & 96.63 & 878.82 & 11.99 & 3.66 \\
\hline HMDB44569 & $\begin{array}{l}\text { TG(16:0/20:5(5Z,8Z,11Z, } \\
14 Z, 17 Z) / 24: 1(15 Z))\end{array}$ & $2.59 \mathrm{E}-07$ & 6.59 & 41.30 & 90.86 & 980.86 & 11.92 & 3.55 \\
\hline HMDB07098 & DG(16:0/16:0/0:0) & $9.62 \mathrm{E}-10$ & 9.02 & 46.10 & 98.60 & 551.50 & 11.45 & 3.55 \\
\hline HMDB56138 & DG(14:1n5/0:0/20:1n9) & $5.88 \mathrm{E}-08$ & 7.23 & 38.30 & 91.85 & 575.50 & 4.65 & 3.52 \\
\hline HMDB07129 & DG(16:1(9Z)/18:0/0:0) & $3.94 \mathrm{E}-08$ & 7.40 & 38.80 & 96.57 & 617.51 & 6.41 & 3.51 \\
\hline LMGP10010881 & PA(18:1(9Z)/16:1(9Z)) & $5.33 \mathrm{E}-08$ & 7.27 & 33.90 & 65.36 & 671.46 & 5.09 & -3.22 \\
\hline LMGP02010655 & $\operatorname{PE}(18: 2(9 Z, 12 Z) / 14: 0)$ & $2.00 \mathrm{E}-05$ & 4.70 & 36.70 & 87.20 & 686.47 & 4.49 & -3.25 \\
\hline HMDB12097 & $\mathrm{SM}(\mathrm{d} 18: 1 / 14: 0)$ & $6.71 \mathrm{E}-09$ & 8.17 & 54.30 & 93.88 & 675.54 & 3.93 & -3.29 \\
\hline HMDB08834 & $\operatorname{PE}(14: 0 / 20: 1(11 Z))$ & $2.01 \mathrm{E}-05$ & 4.70 & 39.50 & 90.78 & 700.53 & 5.03 & -3.39 \\
\hline HMDB60423 & $\begin{array}{l}\text { 7,8-Dihydro-7-hydroxy- } \\
\text { 8-S-glutathionyl- } \\
\text { benzo[a]pyrene }\end{array}$ & $3.97 \mathrm{E}-02$ & 1.40 & 30.50 & 58.63 & 1151.36 & 5.17 & -3.41 \\
\hline HMDB07934 & $\mathrm{PC}(15: 0 / 15: 0)$ & $8.42 \mathrm{E}-09$ & 8.07 & 41.50 & 98.34 & 706.54 & 4.52 & -3.44 \\
\hline HMDB07182 & DG(18:1(11Z)/14:0/0:0) & $1.86 \mathrm{E}-08$ & 7.73 & 38.90 & 97.33 & 549.49 & 4.58 & -3.45 \\
\hline HMDB07103 & DG(16:0/18:2(9Z,12Z)/0:0) & 7.83E-09 & 8.11 & 40.70 & 95.09 & 615.50 & 5.82 & -3.67 \\
\hline LMGP01010459 & $\operatorname{PC}(13: 0 / 15: 0)$ & $1.73 \mathrm{E}-09$ & 8.76 & 40.10 & 91.34 & 678.51 & 4.01 & -3.78 \\
\hline HMDB08002 & $\mathrm{PC}(16: 1(9 \mathrm{Z}) / 16: 1(9 \mathrm{Z}))$ & $1.09 \mathrm{E}-09$ & 8.96 & 48.60 & 91.11 & 730.54 & 4.20 & -4.20 \\
\hline HMDB07998 & $\mathrm{PC}(16: 1(9 \mathrm{Z}) / 14: 0)$ & $1.17 \mathrm{E}-09$ & 8.93 & 36.00 & 82.85 & 702.51 & 4.77 & -4.41 \\
\hline HMDB07932 & $\mathrm{PC}(15: 0 / 14: 0)$ & $6.97 \mathrm{E}-10$ & 9.16 & 37.30 & 84.39 & 690.51 & 5.41 & -4.43 \\
\hline LMGP02010047 & $\operatorname{PE}(18: 3(9 Z, 12 Z, 15 Z) / 16: 0)$ & $9.49 \mathrm{E}-03$ & 2.02 & 35.40 & 84.44 & 714.51 & 4.28 & -4.79 \\
\hline HMDB08828 & $\mathrm{PE}(14: 0 / 18: 1(9 \mathrm{Z}))$ & $4.39 \mathrm{E}-06$ & 5.36 & 42.20 & 69.24 & 688.50 & 4.93 & -4.84 \\
\hline LMGP03010607 & $\begin{array}{l}\operatorname{PS}(20: 3(8 Z, 11 Z, 14 Z) / 19: \\
1(9 Z))\end{array}$ & $1.12 \mathrm{E}-02$ & 1.95 & 40.10 & 96.80 & 870.55 & 4.55 & -5.02 \\
\hline HMDB04884 & $\begin{array}{l}\text { Trihexosylceramide } \\
(\mathrm{d} 18: 1 / 26: 1(17 \mathrm{Z}))\end{array}$ & $9.62 \mathrm{E}-07$ & 6.02 & 32.90 & 66.67 & 1146.82 & 4.69 & -12.62 \\
\hline
\end{tabular}

P-values were obtained from unpaired two tailed t-test. FC means fold-change between the control group and the ZY0511-treated group. The score and isotope similarity were obtained from Progenesis QI software. 
Table III. The top metabolites with fold-change in the HCT116 cell model following ZY0511 treatment.

\begin{tabular}{|c|c|c|c|c|c|c|c|c|}
\hline Compound ID & Compound & P-value & $\log 10 p$ & Score & $\begin{array}{l}\text { Isotope } \\
\text { similarity }\end{array}$ & $\mathrm{m} / \mathrm{z}$ & $\begin{array}{l}\text { Retention } \\
\text { time (min) }\end{array}$ & $\begin{array}{c}\log 2 \\
\text { FC }\end{array}$ \\
\hline HMDB59643 & $\begin{array}{l}\text { 3-Beta-hydroxy-4-beta- } \\
\text { methyl-5-alpha-cholest-7-ene- } \\
\text { 4-alpha-carbaldehyde }\end{array}$ & $2.05 \mathrm{E}-09$ & 8.69 & 44.40 & 86.16 & 429.38 & 2.39 & 3.35 \\
\hline HMDB13407 & PC(o-16:0/20:4(8Z,11Z,14Z,17Z)) & $3.50 \mathrm{E}-05$ & 4.46 & 36.40 & 83.61 & 790.57 & 4.94 & 1.97 \\
\hline HMDB08048 & PC(18:0/20:4(5Z,8Z,11Z,14Z)) & 2.19E-06 & 5.66 & 51.50 & 82.44 & 832.58 & 5.21 & 1.85 \\
\hline HMDB04973 & Glucosylceramide (d18:1/20:0) & $3.45 \mathrm{E}-06$ & 5.46 & 36.50 & 89.03 & 736.61 & 4.52 & 1.80 \\
\hline HMDB59637 & $\begin{array}{l}\text { 3-(3,5-Diiodo-4-hydroxyphenyl) } \\
\text { pyruvate }\end{array}$ & 4.09E-05 & 4.39 & 34.90 & 81.79 & 862.67 & 5.94 & 1.77 \\
\hline HMDB08237 & $\begin{array}{l}\text { PC(18:4(6Z,9Z,12Z,15Z)/ } \\
18: 2(9 Z, 12 Z))\end{array}$ & $1.85 \mathrm{E}-06$ & 5.73 & 53.90 & 89.65 & 778.54 & 4.26 & -1.78 \\
\hline HMDB08826 & $\operatorname{PE}(14: 0 / 18: 0)$ & $6.33 \mathrm{E}-06$ & 5.20 & 37.90 & 92.34 & 692.52 & 4.19 & -1.81 \\
\hline LMGP04030076 & PG(P-20:0/20:1(11Z)) & 9.41E-06 & 5.03 & 37.60 & 89.16 & 815.62 & 3.85 & -1.81 \\
\hline HMDB07780 & $\begin{array}{l}\text { DG(22:6(4Z,7Z,10Z,13Z,16Z, } \\
\text { 19Z)/20:4(8Z,11Z,14Z,17Z)/0:0) }\end{array}$ & $5.74 \mathrm{E}-07$ & 6.24 & 36.80 & 89.59 & 706.54 & 4.43 & -1.86 \\
\hline LMGP02030091 & PE(P-20:0/22:4(7Z,10Z,13Z,16Z)) & $1.27 \mathrm{E}-06$ & 5.90 & 40.70 & 88.83 & 788.59 & 4.47 & -1.89 \\
\hline HMDB10169 & $\operatorname{SM}(d 18: 1 / 16: 0)$ & 4.49E-07 & 6.35 & 38.90 & 94.38 & 702.57 & 4.29 & -1.92 \\
\hline HMDB12095 & $\mathrm{SM}(\mathrm{d} 18: 0 / 24: 1(15 \mathrm{Z}))$ & $8.75 \mathrm{E}-08$ & 7.06 & 46.20 & 88.34 & 832.73 & 9.96 & -1.96 \\
\hline HMDB12097 & $\mathrm{SM}(\mathrm{d} 18: 1 / 14: 0)$ & 8.88E-07 & 6.05 & 49.50 & 85.30 & 675.54 & 3.85 & -1.98 \\
\hline LMGP03030016 & PS(P-16:0/19:0) & $3.40 \mathrm{E}-07$ & 6.47 & 39.30 & 89.79 & 744.55 & 4.39 & -2.01 \\
\hline LMFA03010114 & PGE2alpha dimethyl amine & $3.38 \mathrm{E}-07$ & 6.47 & 27.90 & 45.76 & 733.60 & 3.85 & -2.17 \\
\hline LMGL03014248 & TG(14:0/16:1(9Z)/17:1(9Z))[iso6] & $1.70 \mathrm{E}-08$ & 7.77 & 45.70 & 89.34 & 806.72 & 9.86 & -2.18 \\
\hline HMDB42819 & $\begin{array}{l}\text { TG(14:0/20:4(8Z,11Z,14Z,17Z)/ } \\
\text { 16:1(9Z)) }\end{array}$ & $1.98 \mathrm{E}-07$ & 6.70 & 45.20 & 94.08 & 825.69 & 10.18 & -2.19 \\
\hline LMGL03012631 & TG(12:0/12:0/15:1(9Z))[iso3] & 4.50E-07 & 6.35 & 36.80 & 91.91 & 659.57 & 3.85 & -2.22 \\
\hline LMSP02050006 & $\operatorname{CerP}(\mathrm{d} 18: 1 / 22: 0)$ & $3.63 \mathrm{E}-08$ & 7.44 & 43.00 & 90.34 & 746.57 & 4.67 & -2.28 \\
\hline LMGL03013324 & TG(12:0/16:0/17:1(9Z))[iso6] & $1.86 \mathrm{E}-08$ & 7.73 & 39.80 & 92.81 & 780.70 & 9.79 & -2.32 \\
\hline HMDB07008 & DG(14:0/14:0/0:0) & $1.60 \mathrm{E}-08$ & 7.80 & 54.60 & 98.21 & 495.44 & 10.08 & -2.41 \\
\hline LMGL03013217 & TG(12:0/14:0/18:1(9Z))[iso6] & $7.48 \mathrm{E}-08$ & 7.13 & 51.80 & 96.67 & 766.69 & 9.47 & -2.46 \\
\hline HMDB48568 & TG(16:1(9Z)/14:1(9Z)/18:1(11Z)) & $1.89 \mathrm{E}-08$ & 7.72 & 53.30 & 85.73 & 818.72 & 9.69 & -2.55 \\
\hline LMGL03013351 & TG(12:0/16:1(9Z)/18:1(9Z))[iso6] & $2.66 \mathrm{E}-08$ & 7.58 & 53.90 & 87.71 & 792.71 & 9.57 & -2.66 \\
\hline HMDB42061 & $\mathrm{TG}(14: 0 / 14: 0 / 14: 0)$ & $1.53 \mathrm{E}-07$ & 6.81 & 36.70 & 87.25 & 740.67 & 9.39 & -2.70 \\
\hline HMDB08002 & $\mathrm{PC}(16: 1(9 \mathrm{Z}) / 16: 1(9 \mathrm{Z}))$ & 4.37E-08 & 7.36 & 54.40 & 90.01 & 730.54 & 4.14 & -2.81 \\
\hline HMDB07016 & DG(14:0/18:2(9Z,12Z)/0:0) & $1.61 \mathrm{E}-07$ & 6.79 & 51.50 & 92.84 & 547.47 & 9.65 & -2.83 \\
\hline LMGL02010336 & DG(12:0/18:1(9Z)/0:0)[iso2] & 2.08E-07 & 6.68 & 34.70 & 73.05 & 521.46 & 10.16 & -3.02 \\
\hline HMDB07998 & $\mathrm{PC}(16: 1(9 \mathrm{Z}) / 14: 0)$ & $4.56 \mathrm{E}-08$ & 7.34 & 56.70 & 94.17 & 704.52 & 4.04 & -3.12 \\
\hline LMGL03013464 & $\begin{array}{l}\text { TG(12:0/18:1(9Z)/18:3(9Z,12Z, } \\
\text { 15Z))[iso6] }\end{array}$ & $1.32 \mathrm{E}-08$ & 7.88 & 37.00 & 83.31 & 816.70 & 9.24 & -3.12 \\
\hline LMGL03012619 & TG(13:0/13:0/13:0) & $1.59 \mathrm{E}-05$ & 4.80 & 33.50 & 71.95 & 661.58 & 4.00 & -3.23 \\
\hline LMGP01011245 & $\mathrm{PC}(8: 0 / 20: 0)$ & $1.81 \mathrm{E}-07$ & 6.74 & 38.90 & 90.68 & 678.51 & 3.95 & -3.25 \\
\hline HMDB42752 & TG(14:0/18:3(9Z,12Z,15Z)/15:0) & $1.78 \mathrm{E}-07$ & 6.75 & 51.50 & 81.65 & 804.70 & 9.41 & -3.38 \\
\hline HMDB42271 & TG(14:0/14:1(9Z)/14:0) & $5.32 \mathrm{E}-08$ & 7.27 & 38.40 & 95.33 & 743.61 & 8.81 & -3.78 \\
\hline LMGL03012665 & TG(12:0/16:1(9Z)/16:1(9Z))[iso3] & 2.07E-08 & 7.68 & 48.30 & 87.49 & 769.63 & 8.92 & -3.82 \\
\hline LMGL03012632 & TG(12:0/12:0/16:0)[iso3] & $4.22 \mathrm{E}-07$ & 6.37 & 37.80 & 85.80 & 712.64 & 8.72 & -3.99 \\
\hline HMDB47887 & TG(14:1(9Z)/14:1(9Z)/18:1(11Z)) & $1.50 \mathrm{E}-08$ & 7.82 & 42.50 & 85.85 & 790.69 & 9.09 & -3.99 \\
\hline HMDB07041 & DG(14:1(9Z)/16:1(9Z)/0:0) & $1.94 \mathrm{E}-08$ & 7.71 & 44.00 & 91.73 & 519.44 & 9.65 & -4.00 \\
\hline LMGL03013241 & TG(12:0/14:1(9Z)/16:1(9Z))[iso6] & $1.16 \mathrm{E}-07$ & 6.94 & 41.00 & 81.09 & 741.60 & 8.33 & -4.46 \\
\hline LMGL03013208 & TG(12:0/14:0/14:1(9Z))[iso6] & $3.42 \mathrm{E}-06$ & 5.47 & 39.30 & 97.98 & 715.58 & 8.13 & -5.24 \\
\hline
\end{tabular}

P-values were obtained from unpaired two tailed t-test. FC means fold-change between the control group and the ZY0511-treated group. The score and isotope similarity were obtained from Progenesis QI software. 
A

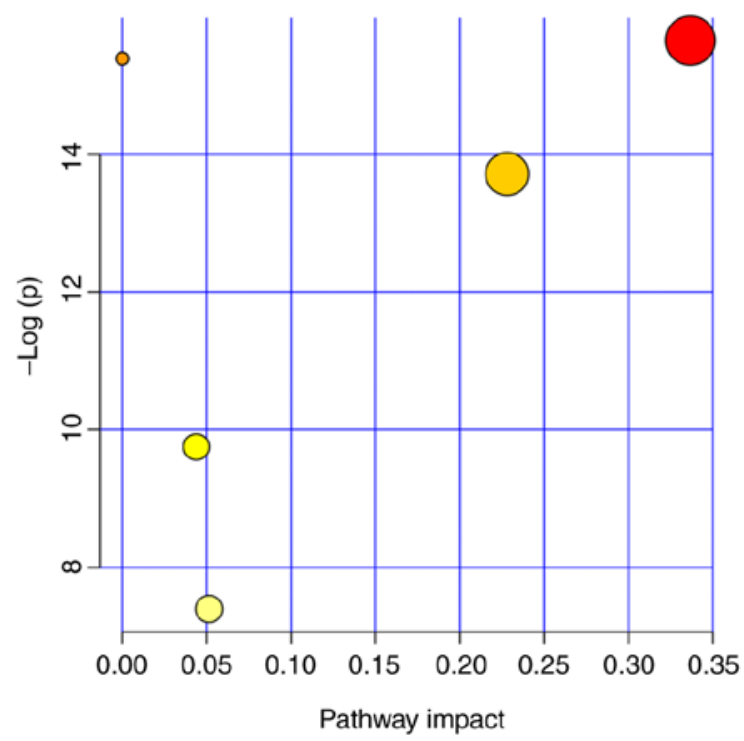

B

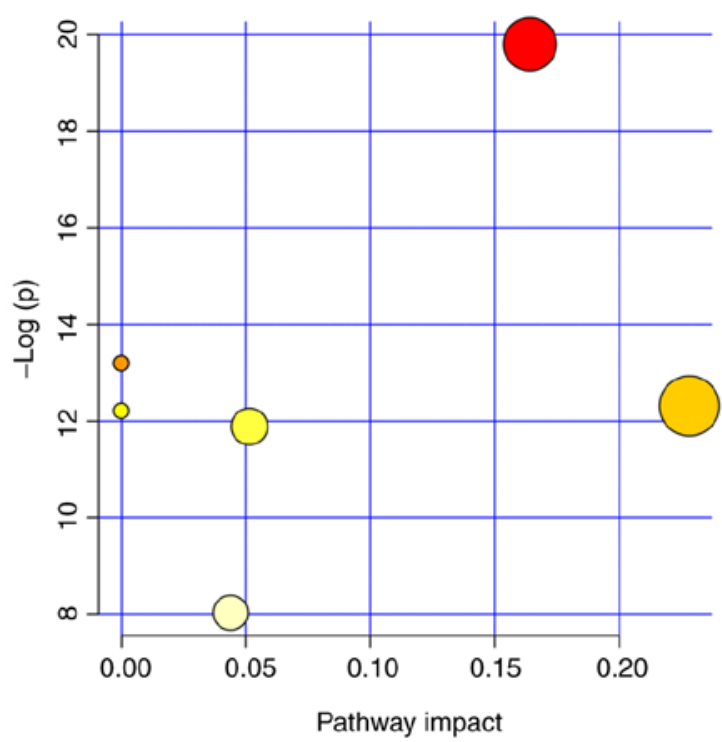

Figure 5. Summary of the pathway analysis by MetaboAnalyst online software in the HeLa model (A) and HCT116 model (B). The further its position is away from the point $(0,0)$, the more significant is the pathway.

and SM (d18:1/26:0) hit the SP metabolism. In HCT116 cells, GluCer (d18:1/20:0), SM (d18:1/16:0) and GalCer (d18:1/16:0) hit the sphingolipid metabolism. Among these, Cer (d18:0/24:1) in HeLa cells was upregulated, while the other metabolites were downregulated. Interestingly, the detailed altered SPs were different which may be attributed to different lipid compositions of these two cell lines.

In addition, we found that the GL metabolism was also modified by ZY0511. The altered metabolites by ZY0511 such as PE (14:0/18:0) and PC (22:0/20:0) in the HCT116 cells are members of the GL metabolism, and these two metabolites were both downregulated. Meanwhile, in HeLa cells, PE (14:0/18:1(11Z)) and PC (18:1(9Z)/18:1(9Z)) hit the GL metabolism either which was obviously downregulated by ZY0511. The detailed information concerning the pathway analysis of the two cell line models are summarized in Tables IV and V. The above suggested lipid metabolism especially SP and GL metabolism were significantly remodeled which suggests that LSD1 may play a critical role in cancer lipid metabolism.

The Cer synthesis process is markedly induced by ZY0511. In both cell line models, SP metabolism was found to be the most modified pathway under the exposure of ZY0511 (Fig. 5). Based on the increased level of Cer and decreased levels of $\mathrm{SM}$ and GluCer, we concluded that the Cer synthesis process was induced by LSD1 inhibition. By qPCR assay, we detected the expression of key Cer synthesis enzymes including SPTL (serine palmitoyltransferase), CERS (ceramide synthases), DEGS (dihydroceramide desaturases) and SMases (sphingomyelinases). We found that expression of ceramide synthases SPTLC1, SPTLC3, CERS2, CERS4, CERS5, CERS6, DEGS1 and DEGS2 were markedly increased by LSD1 inhibition (HeLa-0511 cells compared to HeLa cells and HCT116-0511 cells compared to HCT116 cells) (Fig. 6). Meanwhile, the hydrolysis of SM was also enhanced by the treatment of ZY0511 as the expression of sphingomyelin phosphodiesterase (SMPD1, SMPD2, SMPD3, SMPD4) and ectonucleotide pyrophosphatase/phosphodiesterase family member 7 (ENPP7) were markedly increased (Fig. 6). These results were consistent with the accumulation of Cer and the decrease in SM in lipidomic detection.

Based on the important roles of SP and S1P in tumors, the expression of related metabolic genes was evaluated as well. ZY0511 significantly increased the expression levels of SGPL1 and SGPP1 which catalyze sphingosine production. Inhibition of SGPP1 and SGPL1 was found to cause the accumulation of S1P in cancer cells, promote the invasion of gastric cancer cells (38), and reduce the overall survival of patients (39). Thus, the increasing level of SGPL1 and SGPP1 induced by ZY0511 would benefit cancer therapy.

Next, we explored whether there is a correlation between the expression of metabolic enzymes and LSD1 in tumor patients. The TCGA database was applied to perform the analysis. The results showed that although expression of many proteins were changed after exposure to LSD1 inhibitors, only the expression of SMPD1 and SMPD3 were negatively correlated with LSD1 in tumor samples of patients (Fig. 7) and SMPD1 and SMPD3 may be direct targets of LSD1 in cancer. The induction of other enzymes such as SPTLC1, SPTLC3, CERS2, CERS4, DEGS1, DEGS2, SGPP1 by ZY0511 may not be due to the direct effects of LSD1 inhibition.

Collectively, the cellular Cer production can be induced by LSD1 inhibition. LSD1 may regulate cancer cell survival through modulating the expression of SMPD1 and SMPD3.

\section{Discussion}

Although targeting lysine specific demethylase 1 (LSD1) is a promising strategy for cancer therapy, its underlying mechanism is still poorly understood. In the present study, we performed a thorough lipidomic analysis of human cancer cells by using ZY0511, a novel LSD1 inhibitor as a probe, which 
Table IV. Results from the pathway analysis of the HeLa model.

\begin{tabular}{lcccccc}
\hline Pathways & Total Cmpd & Hits & Raw $p$ & $-\log (\mathrm{p})$ & FDR $p$ & Impact \\
\hline Sphingolipid metabolism & 25 & 4 & $1.591 \mathrm{E}-07$ & 15.654 & $3.623 \mathrm{E}-07$ & 0.33667 \\
Arachidonic acid metabolism & 62 & 1 & $2.070 \mathrm{E}-07$ & 15.391 & $3.623 \mathrm{E}-07$ & 0 \\
Linoleic acid metabolism & 15 & 1 & $2.070 \mathrm{E}-07$ & 15.391 & $3.623 \mathrm{E}-07$ & 0 \\
$\alpha$-linolenic acid metabolism & 29 & 1 & $2.070 \mathrm{E}-07$ & 15.391 & $3.623 \mathrm{E}-07$ & 0 \\
Glycerophospholipid metabolism & 39 & 2 & $1.106 \mathrm{E}-06$ & 13.715 & $1.548 \mathrm{E}-06$ & 0.22806 \\
Glycosylphosphatidylinositol & 14 & 1 & $5.828 \mathrm{E}-05$ & 9.7502 & $6.800 \mathrm{E}-05$ & 0.0439 \\
(GPI)-anchor biosynthesis & & & & & & \\
Glycerolipid metabolism & 32 & 1 & $6.144 \mathrm{E}-04$ & 7.3948 & $6.144 \mathrm{E}-04$ & 0.05145
\end{tabular}

The Total Cmpd is the total number of compounds in the pathway; the Hits is the actually matched number from the user uploaded data; the Raw $p$ is the original P-value calculated from the enrichment analysis; the FDR $p$ is the P-value adjusted using the False Discovery Rate (FDR); the Impact is the pathway impact value calculated from pathway topology analysis.

Table V. Result from pathway analysis of the HCT116 model.

\begin{tabular}{|c|c|c|c|c|c|c|}
\hline Pathways & Total Cmpd & Hits & Raw $p$ & $-\log (p)$ & FDR $p$ & Impact \\
\hline Sphingolipid metabolism & 25 & 3 & $2.510 \mathrm{E}-09$ & 19.802 & 2.010E-08 & 0.16404 \\
\hline Arachidonic acid metabolism & 62 & 1 & $1.860 \mathrm{E}-06$ & 13.196 & $3.710 \mathrm{E}-06$ & 0 \\
\hline Linoleic acid metabolism & 15 & 1 & $1.860 \mathrm{E}-06$ & 13.196 & $3.710 \mathrm{E}-06$ & 0 \\
\hline$\alpha$-Linolenic acid metabolism & 29 & 1 & $1.860 \mathrm{E}-06$ & 13.196 & $3.710 \mathrm{E}-06$ & 0 \\
\hline Glycerophospholipid metabolism & 39 & 2 & $4.500 \mathrm{E}-06$ & 12.311 & $6.640 \mathrm{E}-06$ & 0.22806 \\
\hline Fatty acid metabolism & 50 & 1 & $4.980 \mathrm{E}-06$ & 12.211 & $6.640 \mathrm{E}-06$ & 0 \\
\hline Glycerolipid metabolism & 32 & 1 & $6.890 \mathrm{E}-06$ & 11.885 & $7.880 \mathrm{E}-06$ & 0.05145 \\
\hline $\begin{array}{l}\text { Glycosylphosphatidylinositol } \\
\text { (GPI)-anchor biosynthesis }\end{array}$ & 14 & 1 & $3.260 \mathrm{E}-04$ & 8.0286 & $3.260 \mathrm{E}-04$ & 0.0439 \\
\hline
\end{tabular}

The Total Cmpd is the total number of compounds in the pathway; the Hits is the actually matched number from the user uploaded data; the Raw $p$ is the original P-value calculated from the enrichment analysis; the FDR $p$ is the P-value adjusted using the False Discovery Rate (FDR); the Impact is the pathway impact value calculated from pathway topology analysis.

aimed at a better understanding of the anticancer mechanism of LSD1 inhibitors. We demonstrated that ZY0511 profoundly modified the cancer cell lipidome, especially sphingolipid (SP) metabolism. The increase in Cer and decrease in SM were significantly observed and Cer synthase pathways including de novo synthesis, hydrolysis of SM and the salvage pathway were activated by ZY0511 treatment. These findings were of particular interest which provided a link between LSD1 and cancer cell lipid metabolism especially SP metabolism (Fig. 8). Based on the finding that lipid remodeling is an important alteration in cancer cells, our findings provide a novel view underlying LSD1 regulation of cancer progression.

The incidence and development of tumor is a complex disease process which is affected by many factors, including genetic factors and environmental factors. Cancer cells grow rapidly, divide uncontrollably, and even metastasize from one organ to another. Tumor cells receive various signal stimulation, which is then transmitted to the cell and the nucleus, activating the expression of tumor-related genes, promoting tumor invasion to surrounding tissues and intravascular regeneration. The cell membrane is involved in all these biological processes and is mainly composed of lipids and proteins, among which lipids occupy more than $50 \%$ of the whole cell membrane component (40). In addition, lipids are highly needed to supply energy for the rapid proliferation of tumor cells (41). Lipids such as Cer and SM, are bioactive and act as signal molecules or signal molecule precursors to regulate a variety of cellular functions and biological processes. For example, in the condition of chemotherapy or oxidative stress, the content of Cer and sphingosine increases sharply, inducing cell senescence and even death. Cer was found to induce apoptosis in tumor cells as early as 1993, and is involved in the induction of mitochondrial apoptosis through mitochondrial outer membrane permeabilization which is a possible mechanism for radiation-induced apoptosis (42). In our research, ZY0511 was used as an antitumor small molecule which promoted the accumulation of Cer in tumor cells and induced cell apoptosis as previously reported (43). Meanwhile, various research has indicated that the antitumor effect of LSD1 inhibitors relies on its apoptosis induction ability which furthermore verified the relation between LSD1 and apoptosis $(44,45)$. LSD1 inhibitor JL1037 was found to upregulate pro-apoptotic protein BAX and downregulate anti-apoptotic proteins Bcl-2 and Bcl-XL which cause obvious 

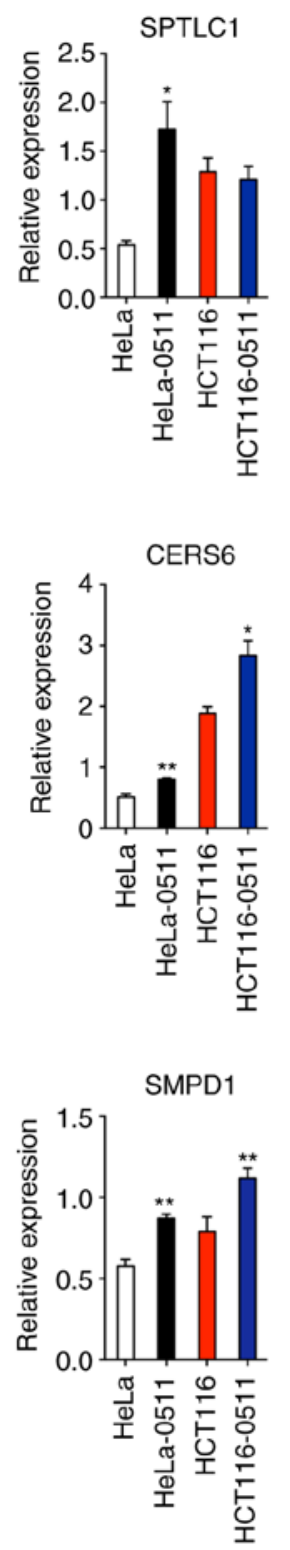
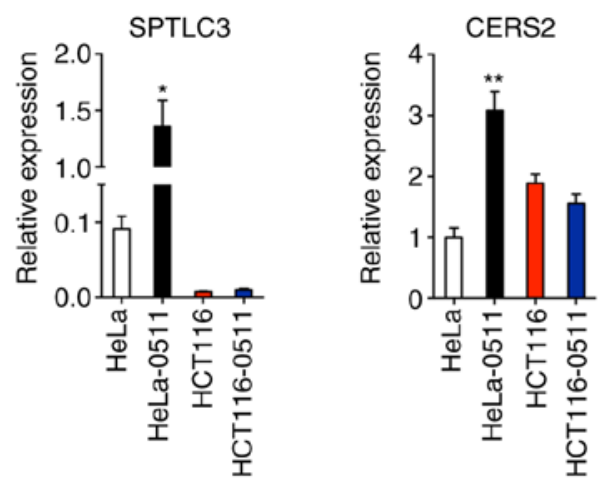

DEGS1

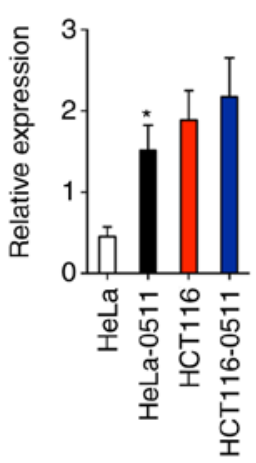

SMPD2
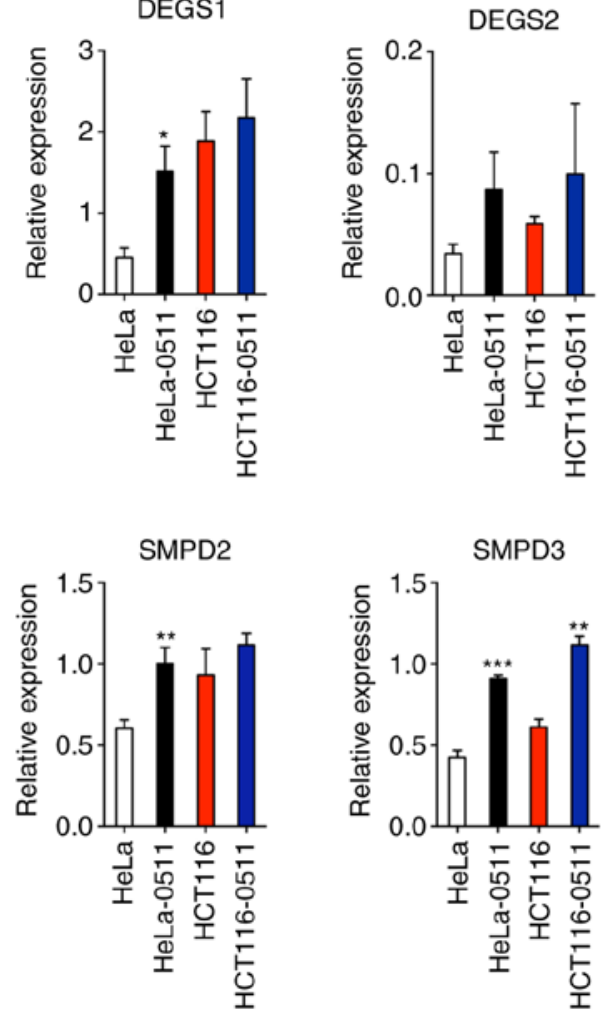
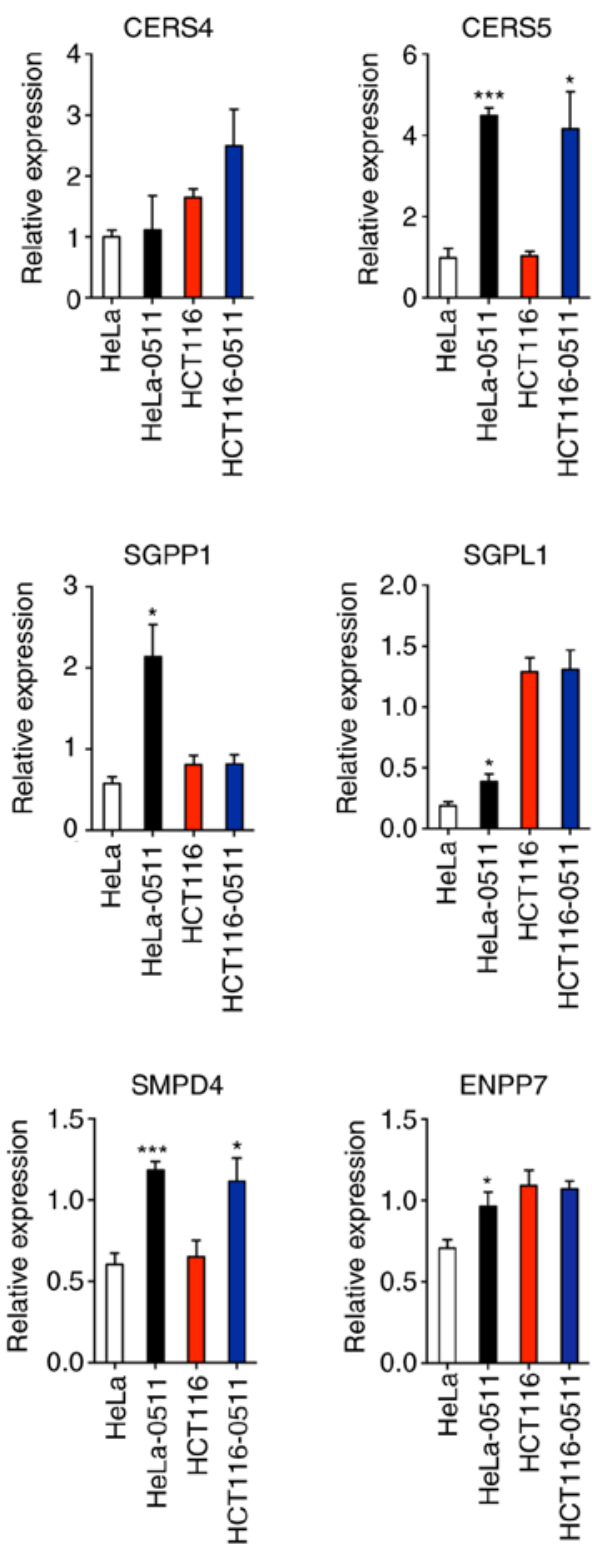

Figure 6. Expression of ceramide synthesis enzymes. The expression of de novo synthesis enzymes (SPTL1, SPTL3, CERS2, CERS4, CERS5, CERS6, DEGS1 and DEGS2), hydrolysis of sphingomyelin enzymes (SMPD1, SMPD2, SMPD3, SMPD4 and ENPP7) and the salvage pathway enzymes (SGPP1 and SGPL1) were determined after ZY0511 exposure in HeLa and HCT116 cells (HeLa-0511 cells compared to HeLa cells and HCT116-0511 cells compared to HCT116 cells). ${ }^{*} \mathrm{P}<0.05,{ }^{* *} \mathrm{P}<0.01,{ }^{* * *} \mathrm{P}<0.001$, based on the unpaired two-tailed Student t-test. SPTL, serine palmitoyltransferase; CERS, ceramide synthase; DEGS, dihydroceramide desaturases; SGPP1, sphingosine-1-phosphate phosphatase 1; SGPL1, sphingosine-1-phosphate lyase 1; SMPD, sphingomyelin phosphodiesterase; ENPP7, ectonucleotide pyrophosphatase/phosphodiesterase family member 7.

apoptosis of cancer cells (42). Our research hints that LSD1 may regulate cell apoptosis through modulating the expression of sphingolipid metabolism genes and there is no research that has previously reported the association between LSD1 and sphingolipid metabolism. Our results provided a novel view of the role of LSD1 in cancer progression and lays the theoretical foundation for LSD1 inhibitor application in the clinic.

In present study, levels of Cer were increased upon the treatment of LSD1 inhibitor. Gutierrez et al showed that Cer induces early apoptosis of human cervical cancer cells by inhibiting reactive oxygen species (ROS) decay, diminishing the intracellular concentration of glutathione and increasing nuclear factor (NF) $-\kappa B$ translocation (46). Cer was also found to contribute to the cellular resistance to doxorubicin including breast, ovary, cervical, and colon cancer cells through upregulation of the gene expression of GCS (47). In colon cancer cells, Cer enhanced FasL-induced cytotoxicity by tumor-specific cytotoxic T lymphocytes (48). With the increase in Cer, we also found a decrease in SM in cells by ZY0511. SM was found to be a chemo-preventive agent in azoxymethane (AOM)-induced colon cancer model of wild-type and $\mathrm{p} 53^{+--}$mice (49). However, SM may play a different role in hypoxic conditions. Klutzny et al found that the inhibition of acid sphingomyelinase in colon cancer caused cellular SM accumulation, which induced cancer cell death specifically in hypoxic tumor spheroids (50).

Because of the critical role of Cer in biological process including cell growth, cancer metastasis and apoptosis, a large number of research studies have focused on the exploration of the role of Cer synthase enzymes in cancer. It has been 
A

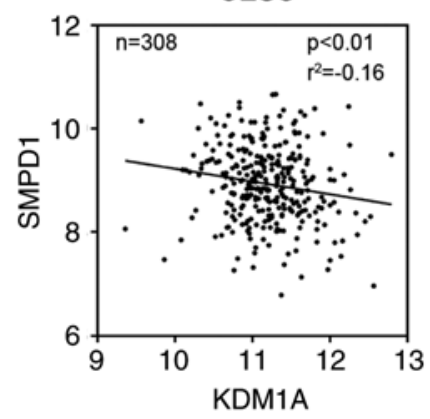

CESC

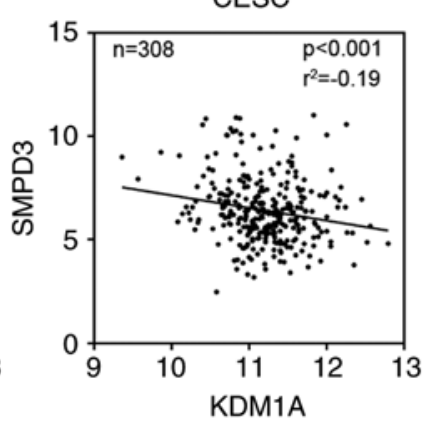

B

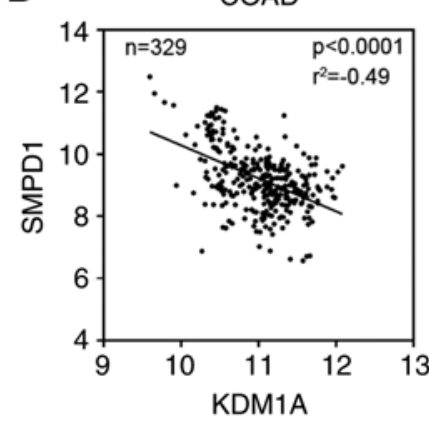

COAD

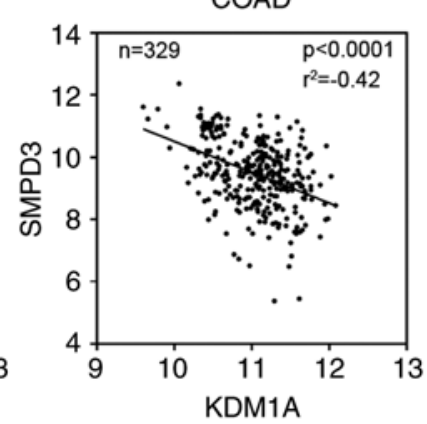

Figure 7. Correlation between gene expression. (A) Correlation between SMPD1, SMPD3 and KDM1A in CESC (cervical squamous cell carcinoma and endocervical adenocarcinoma) patient samples from The Cancer Genome Atlas (TCGA). (B) Correlation between SMPD1, SMPD3 and KDM1A in COAD (colon adenocarcinoma) patient samples from TCGA. SMPD, sphingomyelin phosphodiesterase; KDM1A, lysine-specific demethylase 1 (LSD1).

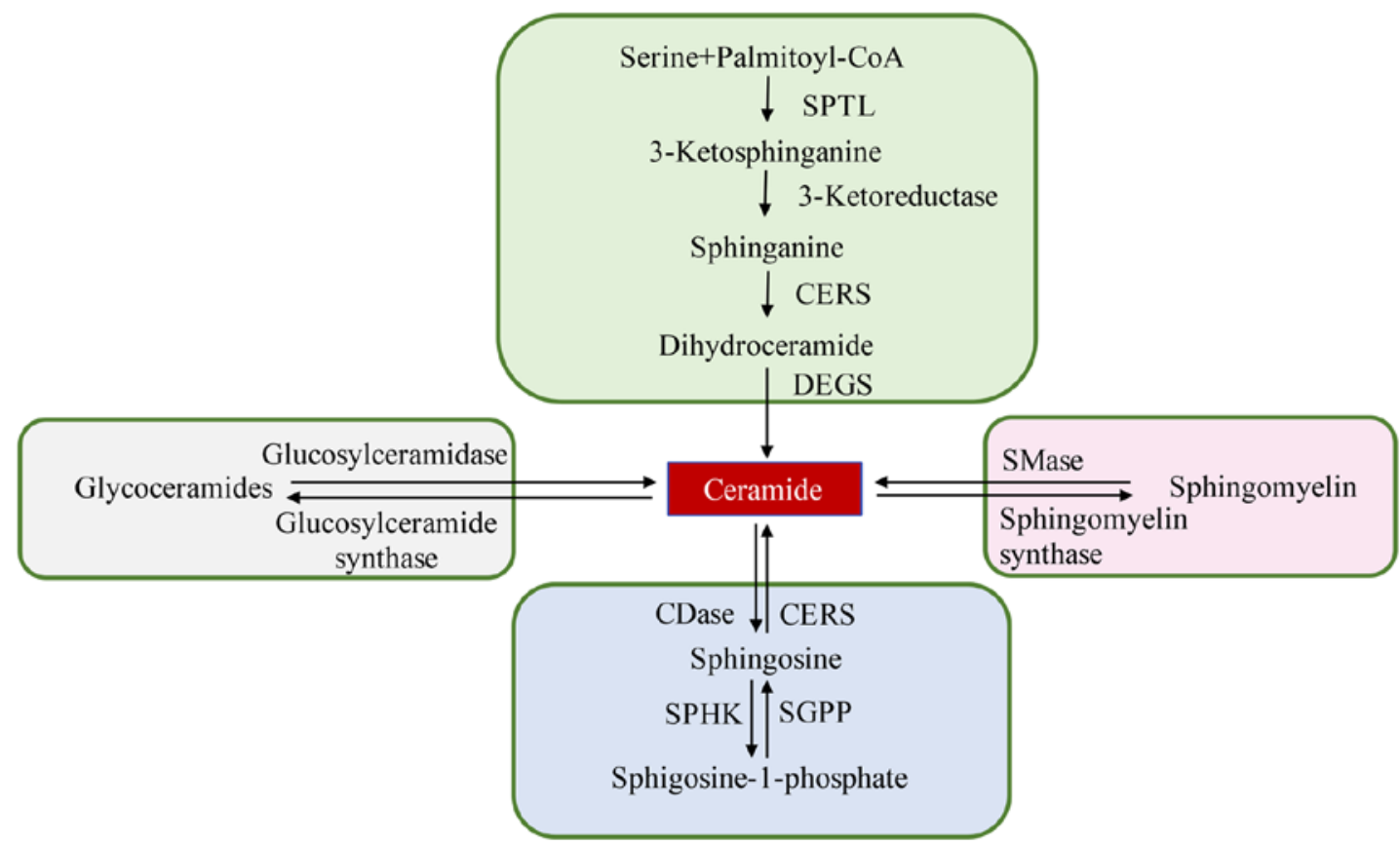

Figure 8. Ceramide synthase pathways in cells and the interconnection between sphingolipid metabolism and bioactive metabolites. CERS, ceramide synthase; SPTL, serine palmitoyltransferase; DEGS, dihydroceramide desaturases; SMase, sphingomyelinase; SPHK, sphingosine kinase; SGPP, sphingosine-1-phosphate phosphatase.

reported that anticancer drugs can upregulate the activity of SMPD1 which catalyzes the production of Cer from SM and promotes cancer cell apoptosis (51). However, SMPDl deficiency was found to reduce tumor development in a manner associated with significant enhancement of Th1-mediated and cytotoxic T-cell-mediated antitumor immunity (52) which suggests the dual role of SMPD1 in different types of tumors. As a Cer-generating enzyme, SMPD3 is implicated in growth arrest, apoptosis and exosome secretion and its deficiency prevents doxorubicin-induced growth arrest (53). In addition, possibly owing to reduced Cer generation, ENPP7 deficiency resulted in increased tumor size and number in mouse models of colon cancer induced by AOM and dextran sulfate sodium (54). In the present study, we demonstrated the upregulation of SMPD1, SMPD2, SMPD3, SMPD4 and ENPP7 after the treatment of ZY0511, most of which act as a tumor suppressor catalyzing the generation of Cer. Most important, we performed gene expression analysis of these genes and LSD1, which showed that SMPDI and SMPD3 may be direct targets of $L S D 1$ as there was a significant negative correlation between these genes and LSD1. Our research for the first time confirmed the regulatory role of LSD1 in the expression of SM hydrolysis genes. We explored the potential upstream regulatory mechanism of SM hydrolysis genes including SMPDI and SMPD3. As SMPD1 and SMPD3 are tumor-suppressor genes, our research provides a novel strategy by which to regulate the SM hydrolysis process through an LSD1 inhibitor. However, the lack of immunoblotting data for the key enzymes including SPTLC1, SPTLC3, CERS2, CERS4, CERS5, CERS6, DEGS1, DEGS2, SMPD1, SMPD2, SMPD3, SMPD4, ENPP7, SGPP1 and SGPL1, is a limitation of the present study which requires further exploration.

Cancer metabolism and epigenetics can be a cause or consequence of malignant transformation. It is now well 
established that cell lipid metabolism and epigenetics interact with each other, and cells exploit this molecular link (55). JMJD3 was identified unexpectedly as a gene-specific transcriptional partner of SIRT1 and activates mitochondrial fatty acid $\beta$-oxidation promoting genes, including $F G F 21$, $C P T 1 A$ and MCAD (56), which suggests the close link between histone demethylation and cell metabolism. Loss of $K D M 4 B$ may impair energy expenditure, adaptive thermogenesis, and adipose tissue lipolysis, resulting in obesity and associated metabolic dysfunction (57). When lysine-specific histone demethylase 2 (LSD2) is lost, proper expression of lipid metabolism genes becomes compromised, leading to an increased susceptibility to toxic cell damage in response to fatty acid exposure (58). As a homologous gene of $L S D 2$, LSDl ablation was found to trigger metabolic reprogramming of brown adipose tissue resulting in the accumulation of diand triacylglycerides (59). In our research, we also found the upregulation of diacylglycerides and triacylglycerides in cancer cells especially HeLa cells after LSD1 inhibition by ZY0511 which is in agreement with a previous study (59). Since the pathway analysis results indicated that the most significantly altered pathway was sphingolipid metabolism, we further investigated the expression of sphingolipid metabolic genes after ZY0511 treatment. The importance of glycerolipids such as di- and triacylglycerides in the process of regulating cancer progression by LSD1 requires further exploration.

In conclusion, we investigated the anticancer mechanism of LSD1 inhibitor ZY0511 from the view of lipid metabolism. The lipidome of cancer cells were significantly modified by ZY0511. We found obvious upregulation of Cer and downregulation of $\mathrm{SM}$ in cancer cells. Our study further confirms the important role of LSD1 in regulating cancer cell sphingolipid metabolism.

\section{Acknowledgements}

Not applicable.

\section{Funding}

This work was supported by Project of the National Natural Sciences Foundation of China (81773198), National Keypoint Research and Invention Program of the China Ministry of Science and Technology (MOST-2016YFC1303200), National S\&T Major project (2018ZX09201018), Science and Technology Innovation Program of Shanxi Provincial Education Department (2020L0177) and Funding for Doctoral Research of Shanxi Province (3C322019039).

\section{Availability of data and materials}

The datasets analyzed during the current study are available in the Mendeley Data repository, https://data.mendeley.com/datasets/vjm2z4vd9j/1.

\section{Authors' contributions}

YZ an XC conceived the concept of the study. YLi, XQ, LT, YLin, SY and HZ performed the experiments. ZZ synthesized compound ZY0511. YLi wrote the paper and conducted all of the experiments. All authors read and approved the manuscript and agree to be accountable for all aspects of the research in ensuring that the accuracy or integrity of any part of the work are appropriately investigated and resolved.

\section{Ethics approval and consent to participate}

Not applicable.

\section{Patient consent for publication}

Not applicable.

\section{Competing interests}

The authors state that they have no competing interests.

\section{References}

1. Shi Y, Lan F, Matson C, Mulligan P, Whetstine JR, Cole PA, Casero RA and Shi Y: Histone demethylation mediated by the nuclear amine oxidase homolog LSD1. Cell 119: 941-953, 2004.

2. Metzger E, Wissmann M, Yin N, Müller JM, Schneider R, Peters AH, GüntherT, Buettner R and Schüle R: LSD1 demethylates repressive histone marks to promote androgen-receptor-dependent transcription. Nature 437: 436-439, 2005.

3. Cai C, He HH, Chen S, Coleman I, Wang H, Fang Z, Chen S, Nelson PS, Liu XS, Brown M and Balk SP: Androgen receptor gene expression in prostate cancer is directly suppressed by the androgen receptor through recruitment of lysine-specific demethylase 1. Cancer Cell 20: 457-471, 2011.

4. Sehrawat A, Gao L, Wang Y, Bankhead A III, McWeeney SK, King CJ, Schwartzman J, Urrutia J, Bisson WH, Coleman DJ, et al: LSD1 activates a lethal prostate cancer gene network independently of its demethylase function. Proc Natl Acad Sci USA 115: E4179-E4188, 2018.

5. Shao G, Wan X, Lai W, Wu C, Jin J, Liu X, Wei Y, Lin Q, Zhang L and Shao Q: Inhibition of lysine-specific demethylase 1 prevents proliferation and mediates cisplatin sensitivity in ovarian cancer cells. Oncol Lett 15: 9025-9032, 2018.

6. Wang Y, Zhang H, Chen Y, Sun Y, Yang F, Yu W, Liang J, Sun L, Yang X, Shi L, et al: LSD1 is a subunit of the NuRD complex and targets the metastasis programs in breast cancer. Cell 138: 660-672, 2009.

7. Hoshino I, Takahashi M, Akutsu Y, Murakami K, Matsumoto Y Suito H, Sekino N, Komatsu A, Iida K, Suzuki T, et al: Genome-wide ChIP-seq data with a transcriptome analysis reveals the groups of genes regulated by histone demethylase LSD1 inhibition in esophageal squamous cell carcinoma cells. Oncol Lett 18: 872-881, 2019.

8. Ding J, Zhang ZM, Xia Y, Liao GQ, Pan Y, Liu S, Zhang Y and Yan ZS: LSD1-mediated epigenetic modification contributes to proliferation and metastasis of colon cancer. Br J Cancer 109: 994-1003, 2013.

9. Schenk T, Chen WC, Gollner S, Göllner S, Howell L, Jin L, Hebestreit K, Klein HU, Popescu AC, Burnett A, et al: Inhibition of the LSD1 (KDM1A) demethylase reactivates the all-transretinoic acid differentiation pathway in acute myeloid leukemia. Nat Med 18: 605-611, 2012.

10. Mohammad HP, Smitheman KN, Kamat CD, Soong D, Federowicz KE, Van Aller GS, Schneck JL, Carson JD, Liu Y, Butticello M, et al: A DNA hypomethylation signature predicts antitumor activity of LSD1 inhibitors in SCLC. Cancer Cell 28: 57-69, 2015.

11. Harris WJ, Huang X, Lynch JT, Spencer GJ, Hitchin JR, Li Y, Ciceri F, Blaser JG, Greystoke BF, Jordan AM, et al: The histone demethylase KDM1A sustains the oncogenic potential of MLL-AF9 leukemia stem cells. Cancer Cell 21: 473-487, 2012.

12. Lim S, Janzer A, Becker A, Zimmer A, Schüle R, Buettner R and Kirfel J: Lysine-specific demethylase 1 (LSD1) is highly expressed in ER-negative breast cancers and a biomarker predicting aggressive biology. Carcinogenesis 31: 512-520, 2010

13. Shi YJ, Matson C, Lan F, Iwase S, Baba T and Shi Y: Regulation of LSD1 histone demethylase activity by its associated factors. Mol Cell 19: 857-864, 2005. 
14. Lee MG, Wynder C, Cooch $\mathrm{N}$ and Shiekhattar R: An essential role for CoREST in nucleosomal histone 3 lysine 4 demethylation. Nature 437: 432-435, 2005.

15. Ketscher A, Jilg CA, Willmann D, Hummel B, Imhof A, Rüsseler V, Hölz S, Metzger E, Müller JM and Schüle R: LSD1 controls metastasis of androgen-independent prostate cancer cells through PXN and LPAR6. Oncogenesis 3: e120, 2014.

16. Liscovitch M and Cantley LC: Lipid second messengers. Cell 77: 329-334, 1994

17. Rabinowitz JD and White E: Autophagy and metabolism. Science 330: 1344-1348, 2010.

18. Huang C and Freter C: Lipid metabolism, apoptosis and cancer therapy. Int J Mol Sci 16: 924-949, 2015.

19. Hannun YA and Bell RM: Lysosphingolipids inhibit protein kinase C: Implications for the sphingolipidoses. Science 235 : 670-674, 1987.

20. Dressler KA, Mathias S and Kolesnick RN: Tumor necrosis factor-alpha activates the sphingomyelin signal transduction pathway in a cell-free system. Science 255: 1715-1718, 1992.

21. Hannun YA and Obeid LM: Principles of bioactive lipid signalling: Lessons from sphingolipids. Nat Rev Mol Cell Biol 9: 139-150, 2008

22. Ogretmen B and Hannun YA: Biologically active sphingolipids in cancer pathogenesis and treatment. Nat Rev Cancer 4: 604-616, 2004.

23. Cuvillier O, Pirianov G, Kleuser B, Vanek PG, Coso OA Gutkind S and Spiegel S: Suppression of ceramide-mediated programmed cell death by sphingosine-1-phosphate. Nature 381: 800-803, 1996

24. Lee MJ, Van Brocklyn JR, Thangada S, Liu CH, Hand AR, Menzeleev R, Spiegel S and Hla T: Sphingosine-1-phosphate as a ligand for the $G$ protein-coupled receptor EDG-1. Science 279: $1552-1555,1998$

25. Sakamoto A, Hino S, Nagaoka $K$, Anan $K$, Takase $R$, Matsumori H, Ojima H, Kanai Y, Arita K and Nakao M: Lysine demethylase LSD1 coordinates glycolytic and mitochondrial metabolism in hepatocellular carcinoma cells. Cancer Res 75 : $1445-1456,2015$.

26. Kosumi K, Baba Y, Sakamoto A, Ishimoto T, Harada K, Nakamura K, Kurashige J, Hiyoshi Y, Iwatsuki M, Iwagami S, et al: Lysine-specific demethylase-1 contributes to malignant behavior by regulation of invasive activity and metabolic shift in esophageal cancer. Int J Cancer 138: 428-439, 2016.

27. Wenk MR: Lipidomics: New tools and applications. Cell 143: 888-895, 2010

28. Zhou Y, Li Y, Wang WJ, Xiang P, Luo XM, Yang L, Yang SY and Zhao YL: Synthesis and biological evaluation of novel (E)-N'-(2,3-dihydro-1H-inden-1-ylidene) benzohydrazides as potent LSD1 inhibitors. Bioorg Med Chem Lett 26: 4552-4557, 2016.

29. Folch J, Lees M and Sloane Stanley GH: A simple method for the isolation and purification of total lipides from animal tissues. J Biol Chem 226: 497-509, 1957.

30. Livak KJ and Schmittgen TD: Analysis of relative gene expression data using real-time quantitative PCR and the 2(-Delta Delta C(T)) method. Methods 25: 402-408, 2001

31. Goldman MJ, Craft B, Hastie M, Repečka K, McDade F, Kamath A, Banerjee A, Luo Y, Rogers D, Brooks AN, et al: Visualizing and interpreting cancer genomics data via the Xena platform. Nat Biotechnol 38: 675-678, 2020.

32. Li Y, Tao L, Zuo Z, Zhou Y, Qian X, Lin Y, Jie H, Liu C, Li Z, Zhang H, et al: ZY0511, a novel, potent and selective LSD1 inhibitor, exhibits anticancer activity against solid tumors via the DDIT4/mTOR pathway. Cancer Lett 454: 179-190, 2019.

33. Birge RB, Boeltz S, Kumar S, Carlson J, Wanderley J, Calianese D, Barcinski M, Brekken RA, Huang X, Hutchins JT, et al: Phosphatidylserine is a global immunosuppressive signal in efferocytosis, infectious disease, and cancer. Cell Death Differ 23: 962-978, 2016

34. Iorio E, Ricci A, Bagnoli M, Pisanu ME, Castellano G, Di Vito M, Venturini E, Glunde K, Bhujwalla ZM, Mezzanzanica D, et al: Activation of phosphatidylcholine cycle enzymes in human epithelial ovarian cancer cells. Cancer Res 70: 2126-2135, 2010.

35. Dolce V, Cappello AR, Lappano R and Maggiolini M: Glycerophospholipid synthesis as a novel drug target against cancer. Curr Mol Pharmacol 4: 167-175, 2011

36. Obeid LM, Linardic CM, Karolak LA and Hannun YA: Programmed cell death induced by ceramide. Science 259 : 1769-1771, 1993
37. Siskind LJ, Feinstein L, Yu T, Davis JS, Jones D, Choi J, Zuckerman JE, Tan W, Hill RB, Hardwick JM and Colombini M: Anti-apoptotic Bcl-2 family proteins disassemble ceramide channels. J Biol Chem 283: 6622-6630, 2008.

38. Oskouian B, Sooriyakumaran P, Borowsky AD, Crans A, Dillard-Telm L, Tam YY, Bandhuvula P and Saba JD: Sphingosine-1-phosphate lyase potentiates apoptosis via $\mathrm{p} 53$ - and p38-dependent pathways and is down-regulated in colon cancer. Proc Natl Acad Sci USA 103: 17384-17389, 2006.

39. Gao XY, Li L, Wang XH, Wen XZ, Ji K, Ye L, Cai J, Jiang WG and Ji JF: Inhibition of sphingosine-1-phosphate phosphatase 1 promotes cancer cells migration in gastric cancer: Clinical implications. Oncol Rep 34: 1977-1987, 2015.

40. Escriba PV, González-Ros JM, Goñi FM, Kinnunen PK, Vigh L, Sánchez-Magraner L, Fernández AM, Busquets X, Horváth I and Barceló-Coblijn G: Membranes: A meeting point for lipids, proteins and therapies. J Cell Mol Med 12: 829-875, 2008

41. Prentki M and Madiraju SR: Glycerolipid metabolism and signaling in health and disease. Endocr Rev 29: 647-676, 2008.

42. Chang KT, Anishkin A, Patwardhan GA, Beverly LJ, Siskind LJ and Colombini M: Ceramide channels: destabilization by Bcl-xL and role in apoptosis. Biochim Biophys Acta 1848: 2374-2384, 2015.

43. Kolesnick R and Fuks Z: Radiation and ceramide-induced apoptosis. Oncogene 22: 5897-5906, 2003.

44. Haydn T, Metzger E, Schuele R and Fulda S: Concomitant epigenetic targeting of LSD1 and HDAC synergistically induces mitochondrial apoptosis in rhabdomyosarcoma cells. Cell Death Dis 8: e2879, 2017.

45. Liu S, Lu W, Li S, Li S, Liu J, Xing Y, Zhang S, Zhou JZ, Xing $\mathrm{H}, \mathrm{Xu} \mathrm{Y}$, et al: Identification of JL1037 as a novel, specific, reversible lysine-specific demethylase 1 inhibitor that induce apoptosis and autophagy of AML cells. Oncotarget 8: 31901-31914, 2017

46. Gutierrez G, Mendoza C, Montano LF and Lopez-Marure R: Ceramide induces early and late apoptosis in human papilloma virus+ cervical cancer cells by inhibiting reactive oxygen species decay, diminishing the intracellular concentration of glutathione and increasing nuclear factor-kappaB translocation. Anticancer Drugs 18: 149-159, 2007.

47. Liu YY, Yu JY, Yin D, Patwardhan GA, Gupta V, Hirabayashi Y, Holleran WM, Giuliano AE, Jazwinski SM, Gouaze-Andersson $\mathrm{V}$, et al: A role for ceramide in driving cancer cell resistance to doxorubicin. FASEB J 22: 2541-2551, 2008.

48. Coe GL, Redd PS, Paschall AV, Lu C, Gu L, Cai H, Albers T, Lebedyeva IO and Liu K: Ceramide mediates FasL-induced caspase 8 activation in colon carcinoma cells to enhance FasL-induced cytotoxicity by tumor-specific cytotoxic T lymphocytes. Sci Rep 6: 30816, 2016.

49. Hu Y, Le Leu RK, Belobrajdic D and Young GP: The potential of sphingomyelin as a chemopreventive agent in AOM-induced colon cancer model: Wild-type and p53+/- mice. Mol Nutr Food Res 52: 558-566, 2008.

50. Klutzny S, Lesche R, Keck M, Kaulfuss S, Schlicker A, Christian S, Sperl C, Neuhaus R, Mowat J, Steckel M, et al: Functional inhibition of acid sphingomyelinase by Fluphenazine triggers hypoxia-specific tumor cell death. Cell Death Dis 8: e2709, 2017.

51. Mizutani N, Omori Y, Kawamoto Y, Sobue S, Ichihara M, Suzuki M, Kyogashima M, Nakamura M, Tamiya-Koizumi K, Nozawa Y and Murate T: Resveratrol-induced transcriptional up-regulation of ASMase (SMPD1) of human leukemia and cancer cells. Biochem Biophys Res Commun 470: 851-856, 2016.

52. Kachler K, Bailer M, Heim L, Schumacher F, Reichel M, Holzinger CD, Trump S, Mittler S, Monti J, Trufa DI, et al: Enhanced acid sphingomyelinase activity drives immune evasion and tumor growth in non-small cell lung carcinoma. Cancer Res 77: 5963-5976, 2017.

53. Shamseddine AA,Clarke CJ,Carroll B, Airola MV,Mohammed S, Rella A, Obeid LM and Hannun YA: P53-dependent upregulation of neutral sphingomyelinase-2: Role in doxorubicin-induced growth arrest. Cell Death Dis 6: e1947, 2015.

54. Chen Y, Zhang P, Xu SC, Yang L, Voss U, Ekblad E, Wu Y, Min Y, Hertervig E, Nilsson $\AA$ and Duan RD: Enhanced colonic tumorigenesis in alkaline sphingomyelinase (NPP7) knockout mice. Mol Cancer Ther 14: 259-267, 2015. 
55. Carrer A and Wellen KE: Metabolism and epigenetics: A link cancer cells exploit. Curr Opin Biotechnol 34: 23-29, 2015.

56. Seok S, Kim YC, Byun S, Choi S, Xiao Z, Iwamori N, Zhang Y, Wang C, Ma J, Ge K, et al: Fasting-induced JMJD3 histone demethylase epigenetically activates mitochondrial fatty acid $\beta$-oxidation. J Clin Invest 128: 3144-3159, 2018.

57. Cheng Y, Yuan Q, Vergnes L, Rong X, Youn JY, Li J, Yu Y, Liu W, Cai H, Lin JD, et al: KDM4B protects against obesity and metabolic dysfunction. Proc Natl Acad Sci USA 115: E5566-E5575, 2018
58. Nagaoka K, Hino S, Sakamoto A, Anan K, Takase R, Umehara T, Yokoyama S, Sasaki Y and Nakao M: Lysine-specific demethylase 2 suppresses lipid influx and metabolism in hepatic cells. Mol Cell Biol 35: 1068-1080, 2015.

59. Duteil D, Tosic M, Lausecker F, Nenseth HZ, Müller JM, Urban S, Willmann D, Petroll K, Messaddeq N, Arrigoni L, et al: Lsd1 ablation triggers metabolic reprogramming of brown adipose tissue. Cell Rep 17: 1008-1021, 2016.

(c) (i)(9) This work is licensed under a Creative Commons Attribution-NonCommercial-NoDerivatives 4.0 International (CC BY-NC-ND 4.0) License. 